Radu Ignat $\cdot$ Felix Otto

\title{
A compactness result in thin-film micromagnetics and the optimality of the Néel wall
}

Received January 29, 2007 and in revised form June 20, 2007

Abstract. We study a model for the magnetization in thin ferromagnetic films. It comes as a variational problem for $S^{1}$-valued maps $m^{\prime}$ (the magnetization) of two variables $x^{\prime}$ :

$$
E_{\varepsilon}\left(m^{\prime}\right)=\varepsilon \int\left|\nabla^{\prime} \cdot m^{\prime}\right|^{2} d x^{\prime}+\left.\left.\frac{1}{2} \int|| \nabla^{\prime}\right|^{-1 / 2} \nabla^{\prime} \cdot m^{\prime}\right|^{2} d x^{\prime} .
$$

We are interested in the behavior of minimizers as $\varepsilon \rightarrow 0$. They are expected to be $S^{1}$-valued maps $m^{\prime}$ of vanishing distributional divergence, $\nabla^{\prime} \cdot m^{\prime}=0$, so that appropriate boundary conditions enforce line discontinuities. For finite $\varepsilon>0$, these line discontinuities are approximated by smooth transition layers, the so-called Néel walls. Néel walls have a line energy density of the order $1 /|\ln \varepsilon|$. One of the main results is that the boundedness of $\left\{|\ln \varepsilon| E_{\varepsilon}\left(m_{\varepsilon}^{\prime}\right)\right\}$ implies the compactness of $\left\{m_{\varepsilon}^{\prime}\right\}_{\varepsilon \downarrow 0}$, so that indeed the limits $m^{\prime}$ will be $S^{1}$-valued and weakly divergence-free. Moreover, we show the optimality of the 1-d Néel wall under 2-d perturbations as $\varepsilon \downarrow 0$.

Keywords. Micromagnetics, Néel wall, compactness, principle of characteristics

\section{Introduction}

In this paper we analyze a 2-d approximation of the micromagnetic energy of a thin film in the absence of external field and crystalline anisotropy. Following [5, 7], the setting is determined by our goal to prove the optimality of Néel walls under 2-d variations. Let $\Omega^{\prime}=(-1,1) \times \mathbb{R}$ be a 2 -d sheet (the cross section of the thin ferromagnetic sample) (see Figure 1). The admissible magnetizations are smooth 2 -d unit-length vector fields

$$
m^{\prime}=\left(m_{1}, m_{2}\right): \mathbb{R}^{2} \rightarrow S^{1}
$$

that macroscopically connect two magnetizations which form an angle (see Figure 2), i.e.,

$$
m^{\prime}\left(x^{\prime}\right)=\left(\begin{array}{c}
m_{1, \infty} \\
\pm \sqrt{1-m_{1, \infty}^{2}}
\end{array}\right) \quad \text { for } \pm x_{1} \geq 1, x_{2} \in \mathbb{R}
$$

R. Ignat: Laboratoire Jacques-Louis Lions, Université Pierre et Marie Curie, B.C. 187, 4 Place Jussieu, 75252 Paris Cedex 05, France; e-mail: ignat@ ann.jussieu.fr

F. Otto: Institute of Applied Mathematics, University of Bonn, Wegelerstrasse 10, 53115 Bonn, Germany; e-mail: otto@iam.uni-bonn.de

Mathematics Subject Classification (2000): Primary 78M35; Secondary 35B65, 35J60, 54D30, $65 \mathrm{M} 25,78 \mathrm{~A} 30$ 


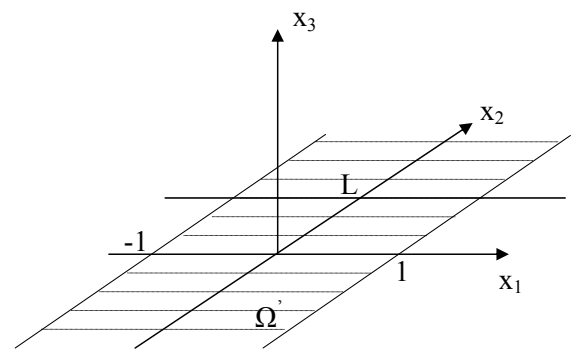

Fig. 1. The infinite domain $\Omega^{\prime}$.
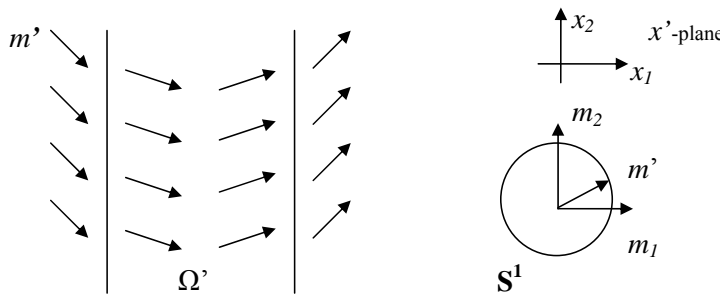

Fig. 2. The admissible magnetization $m^{\prime}$.

where $m_{1, \infty} \in[0,1)$ is some fixed number and we use the shorthand notation $x^{\prime}=$ $\left(x_{1}, x_{2}\right)$. Here and below, the prime always indicates an in-plane quantity. Next to the magnetization $m^{\prime}: \mathbb{R}^{2} \rightarrow S^{1}$, the stray field $h=\left(h_{1}, h_{2}, h_{3}\right): \mathbb{R}^{3} \rightarrow \mathbb{R}^{3}$ is of importance. It is related to the magnetization via the following variational formulation:

$$
\int_{\mathbb{R}^{3}} h \cdot \nabla \zeta d x=\int_{\mathbb{R}^{2}} \zeta \nabla^{\prime} \cdot m^{\prime} d x^{\prime}, \quad \forall \zeta \in C_{c}^{\infty}\left(\mathbb{R}^{3}\right),
$$

where we write $x=\left(x^{\prime}, x_{3}\right) \in \mathbb{R}^{3}$ and $\nabla^{\prime} \cdot m^{\prime}$ for the in-plane divergence of $m^{\prime}$. Classically, this is,

$$
\begin{cases}\nabla \cdot h=0 & \text { in } \mathbb{R}^{3} \backslash\left(\mathbb{R}^{2} \times\{0\}\right), \\ {\left[h_{3}\right]=-\nabla^{\prime} \cdot m^{\prime}} & \text { on } \mathbb{R}^{2} \times\{0\},\end{cases}
$$

where $\left[h_{3}\right]$ denotes the jump of the out-of-plane component of $h$ across the plane $\mathbb{R}^{2} \times\{0\}$.

The micromagnetic model states that the experimentally observed ground state for the magnetization $\mathrm{m}^{\prime}$ and for the stray field is a (local) minimizer of the micromagnetic energy. In order to assign the energy density to this configuration we assume that

$$
m^{\prime} \text { and } h \text { are } L \text {-periodic in the infinite } x_{2} \text {-direction, }
$$

where $L$ is an arbitrary positive number. In this paper we focus on the following nondimensionalized energy functional:

$$
E_{\varepsilon}\left(m^{\prime}, h\right)=\varepsilon \int_{\mathbb{R} \times[0, L)}\left|\nabla^{\prime} \cdot m^{\prime}\right|^{2} d x^{\prime}+\int_{\mathbb{R} \times[0, L) \times \mathbb{R}}|h|^{2} d x,
$$


where $\varepsilon>0$ is a small parameter. In fact, $\varepsilon$ is a non-dimensional quantity formed from three length scales: a material length scale, the film thickness and the film width (see [5, 7]). The first term in (5) comes from the exchange energy (in fact, it is smaller than the usual exchange energy term represented by the Dirichlet integral of $m^{\prime}$ ), and the energy of the stray field is also called the magnetostatic energy. Notice that the stray field $h$ can be minimized out. Given $m^{\prime}$, it is characterized by (2) respectively (3) and $\nabla \times h=0$. We thus recover the static part of the Maxwell equations. In particular, $h=-\nabla u$, where the potential $u$ is characterized by

$$
\begin{cases}\Delta u=0 & \text { in } \mathbb{R}^{3} \backslash\left(\mathbb{R}^{2} \times\{0\}\right), \\ {\left[\frac{\partial u}{\partial x_{3}}\right]=\nabla^{\prime} \cdot m^{\prime}} & \text { on } \mathbb{R}^{2} \times\{0\} .\end{cases}
$$

Rewriting this in Fourier space, we see that the stray field energy is given by the homogeneous $H^{-1 / 2}$-norm of $\nabla^{\prime} \cdot m^{\prime}$ :

$$
\min _{h \text { with [2] }} \int_{\mathbb{R} \times[0, L) \times \mathbb{R}}|h|^{2} d x=\left.\left.\frac{1}{2} \int_{\mathbb{R} \times[0, L)}|| \nabla^{\prime}\right|^{-1 / 2} \nabla^{\prime} \cdot m^{\prime}\right|^{2} d x^{\prime} .
$$

In view of (6) and the electrostatic analogy, one thinks of $\nabla^{\prime} \cdot m^{\prime}$ as a "magnetic charge density". Hence, the energy forces magnetizations with small charge density $\nabla^{\prime} \cdot m^{\prime}$, a principle which is called "pole avoidance". Now we shall informally explain how the principle of pole avoidance leads to the formation of walls (i.e., transition layers). For this discussion, we neglect the first term in (5). For simplicity, we assume that the mesoscopic transition angle imposed by (1) on the boundary $\partial \Omega^{\prime}$ is $180^{\circ}$, i.e., $m^{\prime} \cdot v^{\prime}=0$ on $\partial \Omega^{\prime}$. The boundary effects in the tangential direction are excluded by our choice of $\Omega^{\prime}$ which is infinite in the $x_{2}$-direction. The magnetostatic energy will try to enforce the divergencefree condition for $m^{\prime}$, i.e., $\nabla^{\prime} \cdot m^{\prime}=0$ in $\Omega^{\prime}$. Therefore, we arrive at

$$
\left|m^{\prime}\right|=1 \text { and } \nabla^{\prime} \cdot m^{\prime}=0 \text { in } \Omega^{\prime} .
$$

We notice that the conditions in (7) are too rigid for smooth magnetization $m^{\prime}$. This can be seen by writing $m^{\prime}=\nabla^{\prime} \downarrow$ with the help of a "stream function" $\psi$. Then (7) implies that $\psi$ is a solution of the Dirichlet problem for the eikonal equation:

$$
\left|\nabla^{\prime} \psi\right|=1 \quad \text { in } \Omega^{\prime} .
$$

Using the method of characteristics, it follows that there is no smooth solution of the equation (8) such that $m^{\prime}$ satisfies the boundary conditions (1). On the other hand, there are many continuous solutions that satisfy (8) away from a set of vanishing Lebesgue measure. One of them is the "viscosity solution" given by the distance function

$$
\psi\left(x^{\prime}\right)=\operatorname{dist}\left(x^{\prime}, \partial \Omega^{\prime}\right)
$$

that corresponds to the so-called Landau state for the magnetization $m^{\prime}$ (see Figure 3.) Hence, the divergence-free equation in (7) has to be interpreted in the distribution sense and the boundary conditions (1) are expected to induce line singularities for solutions $\mathrm{m}^{\prime}$. 


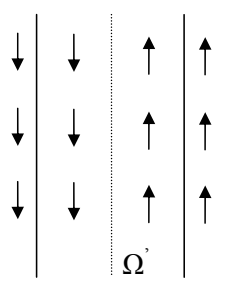

Fig. 3. Landau state in $\Omega^{\prime}$.

These ridges ("ridges" from the point of view of $\psi$ ) are an idealization of walls in thinfilm elements at the mesoscopic level. At the microscopic level, they are replaced by smooth transition layers where the magnetization varies very quickly on a small length scale, which we will address below. A final remark is that the normal component of $\mathrm{m}^{\prime}$ does not jump across these discontinuity lines (because of (7)), and therefore the normal of the mesoscopic wall is determined by the angle between the mesoscopic levels in the adjacent domains.

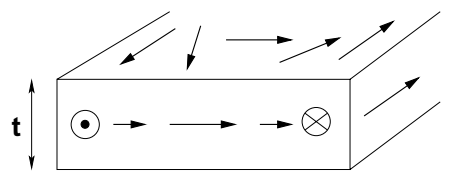

Fig. 4. Néel wall in a 3-d cylinder.

We now take a closer look at the transition layer itself, which is called the Néel wall in the micromagnetics jargon (see Figure 4). As usual, one first considers 1-d transition layers, i.e.,

$$
m^{\prime}=\left(m_{1}\left(x_{1}\right), m_{2}\left(x_{1}\right)\right) .
$$

Notice that the continuous transition layers are necessarily not charge-free,

$$
\nabla^{\prime} \cdot m^{\prime}=\frac{d m_{1}}{d x_{1}} \neq 0
$$

Hence there is a competition between the first and the second term in (5). The prototype is the $180^{\circ}$ Néel wall which corresponds to the boundary conditions (1) for $m_{1, \infty}=0$, i.e.,

$$
m^{\prime}\left(x_{1}\right)=\left(\begin{array}{l}
0 \\
\pm 1
\end{array}\right) \quad \text { for } \pm x_{1} \geq 1
$$

Let us now discuss the scaling of the energy of the prototypical Néel wall. For magnetizations (9), the specific energy (5) reduces to

$$
E_{\varepsilon}^{1 d}\left(m^{\prime}\right)=\varepsilon \int_{\mathbb{R}}\left|\frac{d m_{1}}{d x_{1}}\right|^{2} d x_{1}+\left.\left.\frac{1}{2} \int_{\mathbb{R}}|| \frac{d}{d x_{1}}\right|^{1 / 2} m_{1}\right|^{2} d x_{1} .
$$


We define the Néel wall as the 1-d minimizer of (11) under the boundary constraint (10). The Néel wall is a two-length-scale object: a small core $\left(\left|x_{1}\right| \lesssim w_{\text {core }}\right)$ with fast varying rotation and a logarithmically decaying tail $\left(w_{\text {core }} \lesssim\left|x_{1}\right| \lesssim 1\right)$. The finiteness of $\Omega^{\prime}$ in the $x_{1}$-direction in our setting serves as the confining mechanism for the Néel wall tail. This two-scale structure permits the Néel wall to decrease the specific energy by a logarithmic factor. The prediction of the logarithmic decay was formally proved by Riedel and Seeger [13]; a detailed mathematical discussion of their results was carried out by García-Cervera [8]. Finally, Melcher [11, 12] rigorously established the exact logarithmic scaling for the $180^{\circ}$ Néel wall tails: The minimizer $m_{1}$ with $m_{1}(0)=1$ is symmetric around $0\left(w_{\text {core }} \sim \varepsilon\right)$ and satisfies

$$
m_{1}\left(x_{1}\right) \sim \frac{\ln \frac{1}{\left|x_{1}\right|}}{|\ln \varepsilon|} \quad \text { for } \varepsilon \lesssim\left|x_{1}\right| \lesssim 1
$$

(see Figure 5). Moreover, the leading order term of the minimal energy level is

$$
\min _{\text {99, } 10,} E_{\varepsilon}^{1 d}\left(m^{\prime}\right) \approx \frac{\pi}{2|\ln \varepsilon|} \text { for } \varepsilon \ll 1 \text {. }
$$

The stability of $180^{\circ}$ Néel walls under arbitrary 2-d modulation was proved by DeSimone, Knüpfer and Otto [5]:

$$
\min _{m_{m^{\prime}, h}} E_{\varepsilon}\left(m^{\prime}, h\right) \approx \min _{m^{\prime}=m^{\prime}\left(x_{1}, x_{2}\right) \text { with } 100} E_{\varepsilon}\left(m^{\prime}, h\right) \approx \frac{\pi L}{2|\ln \varepsilon|} \quad \text { for } \varepsilon \ll 1 .
$$
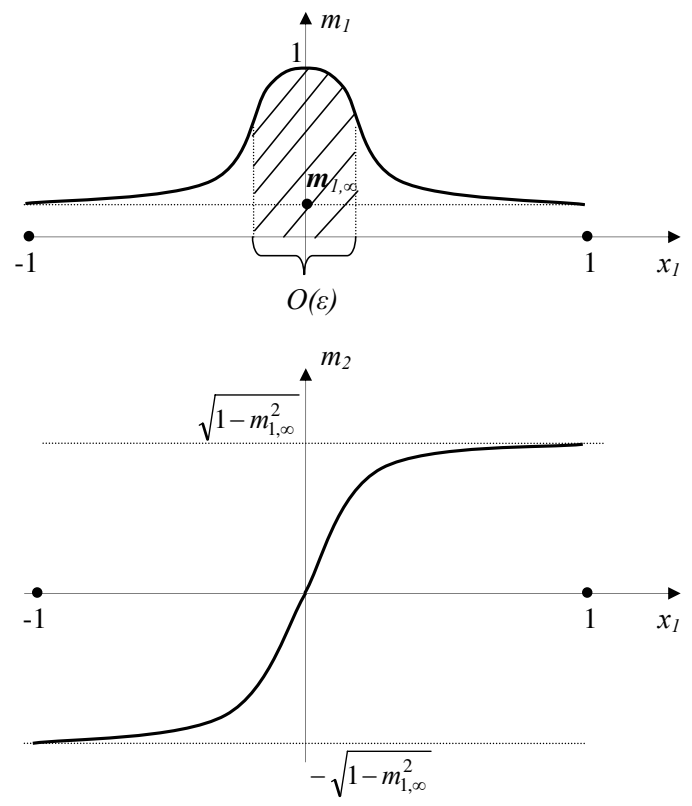

Fig. 5. Qualitative behavior of the Néel wall of angle $2 \arccos m_{1, \infty}$. 
This means that asymptotically, the minimal energy $E_{\varepsilon}$ is assumed by a straight wall. More precisely, the variations of the optimal 1-d transition layer in the $x_{2}$-direction will not decrease the leading order term in the energy.

Our first result is a qualitative property of the optimal 1-d transition layers: We prove that, asymptotically, the minimal energy can be assumed only by the straight walls. This property holds for general boundary conditions (1). It is based on a compactness result for magnetizations $\left\{m_{\varepsilon}^{\prime}\right\}_{\varepsilon \downarrow 0}$ with energies $E_{\varepsilon}$ close to the minimal energy level: Any accumulation limit $m^{\prime}$ has the singularities concentrated on a vertical line (see Figure 6.

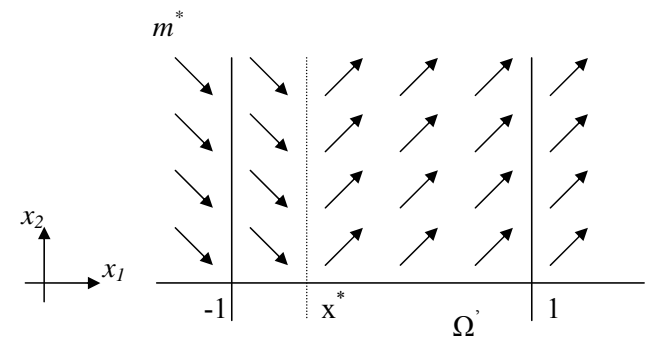

Fig. 6. Straight wall.

Theorem 1. Let $m_{1, \infty} \in[0,1)$ and $L>0$ be given. For any $\delta>0$ there exists $\varepsilon_{0}>0$ with the following property: Given $m^{\prime}: \mathbb{R}^{2} \rightarrow S^{1}$ and $h: \mathbb{R}^{3} \rightarrow \mathbb{R}^{3}$ such that

- $m^{\prime}$ and $h$ are L-periodic in $x_{2}$, i.e., (4) holds,

- $m^{\prime}$ satisfies the boundary conditions (1),

- $m^{\prime}$ and $h^{\prime}$ are related by (2),

- $|\ln \varepsilon| E_{\varepsilon}\left(m^{\prime}, h\right) \leq \frac{1}{2} \pi L\left(1-m_{1, \infty}\right)^{2}+\varepsilon_{0}$ for some $0<\varepsilon \leq \varepsilon_{0}$,

we have

$$
\int_{\mathbb{R} \times[0, L)}\left|m^{\prime}-m^{*}\right| d x^{\prime} \leq \delta,
$$

where $m^{*}$ is a straight wall given by

$$
m^{*}\left(x_{1}, x_{2}\right)=\left(\begin{array}{c}
m_{1, \infty} \\
\pm \sqrt{1-m_{1, \infty}^{2}}
\end{array}\right) \quad \text { for } \pm x_{1}> \pm x_{1}^{*}
$$

for some $x_{1}^{*} \in[-1,1]$

Remark 1. The estimate (13) also holds in $L^{p}$ for any $\delta_{p}>0$ and $1 \leq p<\infty$.

Let us first discuss the compactness result for the case of 1-d magnetizations. We are interested in the asymptotics as $\varepsilon \rightarrow 0$ of families of 1-d magnetizations in the more general context of an energy regime $O(1 /|\ln \varepsilon|)$. We show that such a sequence of magnetizations is relatively compact in $L_{\text {loc }}^{1}$ and the accumulation points in $L_{\text {loc }}^{1}$ concentrate 


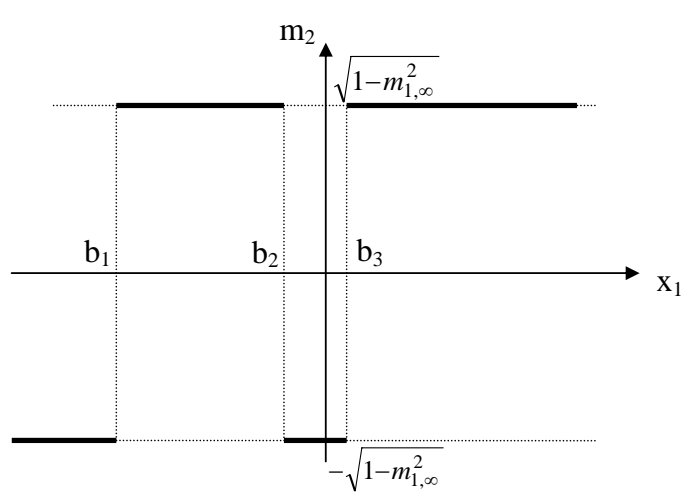

Fig. 7. The $m_{2}$ component of a limit with three walls.

on a finite number of walls (see Figure 7). As a direct consequence, we obtain the optimality of the straight walls over 1-d perturbations in the asymptotic regime of the minimal energy.

Theorem 2. Let $m_{1, \infty} \in[0,1)$. Consider a sequence $\left\{\varepsilon_{k}\right\}_{k \in \mathbb{N}} \subset(0, \infty)$ with $\varepsilon_{k} \downarrow 0$. For $k \in \mathbb{N}$, let $m_{k}^{\prime}=\left(m_{1, k}, m_{2, k}\right): \mathbb{R} \rightarrow S^{1}$ be such that (1) holds and

$$
\limsup _{k \rightarrow \infty}\left|\ln \varepsilon_{k}\right|\left(\varepsilon_{k} \int_{\mathbb{R}}\left|\frac{d m_{1, k}}{d x_{1}}\right|^{2} d x_{1}+\left.\left.\frac{1}{2} \int_{\mathbb{R}}|| \frac{d}{d x_{1}}\right|^{1 / 2} m_{1, k}\right|^{2} d x_{1}\right)<\infty
$$

Then $\left\{m_{k}^{\prime}\right\}_{k \uparrow \infty}$ is relatively compact in $L_{\text {loc }}^{1}(\mathbb{R})$. Moreover, any accumulation point $m^{\prime}$ : $\mathbb{R} \rightarrow S^{1}$ of the sequence $\left\{m_{k}^{\prime}\right\}_{k \uparrow \infty}$ in $L_{\text {loc }}^{1}$ is of bounded variation and can be written as

$$
m^{\prime}=\sum_{n=1}^{2 N}\left(\begin{array}{c}
m_{1, \infty} \\
(-1)^{n} \sqrt{1-m_{1, \infty}^{2}}
\end{array}\right) 1_{\left(b_{n-1}, b_{n}\right)}
$$

where $-\infty=b_{0}<b_{1}<\cdots<b_{2 N-1}<b_{2 N}=+\infty$ and $b_{n} \in[-1,1]$ for $n=$ $1, \ldots, 2 N-1$.

One may ask whether the above sequences of 1-d magnetizations are relatively compact in $B V$ since their limit has bounded variation. The answer is negative in general. For that, we construct a family of 1-d magnetizations with the energy level in the regime $O(1 /|\ln \varepsilon|)$ such that the total variations of $\left\{m_{1, k}\right\}$ blow up as $k \rightarrow \infty$ :

Theorem 3. There exists a sequence $\left\{m_{k}^{\prime}: \mathbb{R} \rightarrow S^{1}\right\}_{k \in \mathbb{N}}$ with the properties:

- (1) holds for some $m_{1, \infty} \in[0,1)$,

- $\lim _{k \rightarrow \infty} \int_{\mathbb{R}}\left|\frac{d m_{1, k}}{d x_{1}}\right| d x_{1}=\infty$,

- 15 holds for some $\left\{\varepsilon_{k}\right\}_{k \uparrow \infty}$ with $\varepsilon_{k} \rightarrow 0$. 
Now we investigate the asymptotics as $\varepsilon \rightarrow 0$ of families of 2-d magnetizations when the energy $E_{\varepsilon}\left(m_{\varepsilon}^{\prime}, h_{\varepsilon}\right)$ is in the regime $O(1 /|\ln \varepsilon|)$. One of the issues we discuss here is the question of the $L_{\text {loc }}^{1}$-compactness of the magnetizations $\left\{m_{\varepsilon}^{\prime}\right\}_{\varepsilon \downarrow 0}$ in the above energy regime, i.e., whether the topological constraint $\left|m_{\varepsilon}^{\prime}\right|=1$ passes to the limit. The difficulty arises from the fact that in general the sequence of divergences $\left\{\nabla^{\prime} \cdot m_{\varepsilon}^{\prime}\right\}$ is not uniformly bounded in $L^{1}$ (a counter-example is given in Theorem 3). This was one of the particularities used in the entropy methods for proving compactness results for Modica-Mortola type problems; we refer to the studies of Jin and Kohn [10], Ambrosio, De Lellis and Mantegazza [3], DeSimone, Kohn, Müller and Otto [6], Alouges, Rivière and Serfaty [2], Rivière and Serfaty [14], and Jabin, Otto and Perthame [9]. For our model, the idea is to use a duality argument in the spirit of [5] based on an $\varepsilon$-perturbation of a logarithmically failing Gagliardo-Nirenberg inequality (see Section 2). Since the compactness result is a local issue, we state it in the context of the unit ball $B_{1} \subset \mathbb{R}^{3}$ with no boundary conditions imposed:

Theorem 4. Consider a sequence $\left\{\varepsilon_{k}\right\}_{k \in \mathbb{N}} \subset(0, \infty)$ with $\varepsilon_{k} \downarrow 0$. For $k \in \mathbb{N}$, let $m_{k}^{\prime}$ : $B_{1}^{\prime} \rightarrow S^{1}$ and $h_{k}: B_{1} \rightarrow \mathbb{R}^{3}$ be related by

$$
\int_{B_{1}} h_{k} \cdot \nabla \zeta d x=\int_{B_{1}^{\prime}} \zeta \nabla^{\prime} \cdot m_{k}^{\prime} d x^{\prime}, \quad \forall \zeta \in C_{c}^{\infty}\left(B_{1}\right) .
$$

Suppose that

$$
\limsup _{k \rightarrow \infty}\left|\ln \varepsilon_{k}\right|\left(\varepsilon_{k} \int_{B_{1}^{\prime}}\left|\nabla^{\prime} \cdot m_{k}^{\prime}\right|^{2} d x^{\prime}+\int_{B_{1}}\left|h_{k}\right|^{2} d x\right)<\infty .
$$

Then $\left\{m_{k}^{\prime}\right\}_{k \uparrow \infty}$ is relatively compact in $L^{1}\left(B_{1}^{\prime}\right)$ and any accumulation point $m^{\prime}: B_{1}^{\prime} \rightarrow$ $\mathbb{R}^{2}$ satisfies

$$
\left|m^{\prime}\right|=1 \quad \text { a.e. in } B_{1}^{\prime} \quad \text { and } \quad \nabla^{\prime} \cdot m^{\prime}=0 \text { distributionally in } B_{1}^{\prime} \text {. }
$$

We now focus on the behavior of the finite-energy states $m^{\prime}$. As in 8 , we formally find from (18) that $m^{\prime}=\nabla^{\prime \perp} \psi$ where $\psi$ satisfies the eikonal equation $\left|\nabla^{\prime} \psi\right|=1$. We discuss the case of zero-energy states, i.e., $m^{\prime}$ is an accumulation point of sequences $\left\{m_{\varepsilon}^{\prime}\right\}_{\varepsilon \downarrow 0}$ such that the limit in (17) vanishes for some stray fields $\left\{h_{\varepsilon}\right\}$ (in the absence of any boundary conditions). We show that every zero-energy state $m^{\prime}$ is locally Lipschitz continuous. The main tool is the principle of characteristics for the eikonal equation. The difference with respect to the zero-energy states for the Ginzburg-Landau models treated in [9] consists in the avoidance of vortices. Our result can be stated as follows:

Theorem 5. Consider a sequence $\left\{\varepsilon_{k}\right\}_{k \in \mathbb{N}} \subset(0, \infty)$ with $\varepsilon_{k} \downarrow 0$. For $k \in \mathbb{N}$, let $m_{k}^{\prime}$ : $B_{1}^{\prime} \rightarrow S^{1}$ and $h_{k}: B_{1} \rightarrow \mathbb{R}^{3}$ be related by 16 . Suppose that

$$
\lim _{k \rightarrow \infty}\left|\ln \varepsilon_{k}\right|\left(\varepsilon_{k} \int_{B_{1}^{\prime}}\left|\nabla^{\prime} \cdot m_{k}^{\prime}\right|^{2} d x^{\prime}+\int_{B_{1}}\left|h_{k}\right|^{2} d x\right)=0 .
$$


Then any accumulation point $m^{\prime}: B_{1}^{\prime} \rightarrow \mathbb{R}^{2}$ of $\left\{m_{k}^{\prime}\right\}_{k \uparrow \infty}$ in $L^{1}\left(B_{1}^{\prime}\right)$ satisfies

(a) $m^{\prime}$ is locally Lipschitz in $B_{1}^{\prime}$;

(b) $m^{\prime}$ satisfies the principle of characteristics related to $\left[18\right.$, i.e., for any $x_{0}^{\prime} \in B_{1}^{\prime}$ we have

$$
m^{\prime}\left(x_{0}^{\prime}+t m^{\prime}\left(x_{0}^{\prime}\right)^{\perp}\right)=m^{\prime}\left(x_{0}^{\prime}\right) \quad \text { for any } t \in \mathbb{R} \text { with } x_{0}^{\prime}+t m^{\prime}\left(x_{0}^{\prime}\right)^{\perp} \in B_{1}^{\prime}
$$

(see Figure 8 .

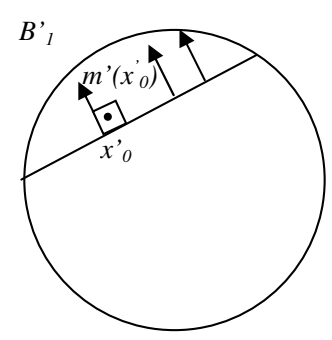

Fig. 8. Principle of characteristics.

Remark 2. In general, a function $m^{\prime}$ satisfying (a) and (b) in Theorem 5 is not globally Lipschitz in $B_{1}^{\prime}$; an example is given by

$$
m^{\prime}\left(x^{\prime}\right)=\left(\frac{x^{\prime}-P}{\left|x^{\prime}-P\right|}\right)^{\perp} \quad \text { for any } x^{\prime} \in B_{1}^{\prime},
$$

for some $P \in \partial B_{1}^{\prime}$ ( $P$ plays the role of a vortex on the boundary).

The outline of the paper is as follows. In Section 2, we give some fundamental estimates based on a duality argument and a logarithmically failing interpolation inequality. In Section 3, we prove Theorem 4 In Section 4, we focus on the zero-energy states: We establish a list of lemmas that lead to Theorem 5 In Section 5, we show the optimality of the straight walls in Theorem 1 as an application of Theorems 4 and 5 . In Section 6 we discuss the behavior of 1-d magnetizations by proving Theorems 2 and 3 .

\section{Some fundamental localized estimates}

We present some inequalities in the spirit of [5] that are to be used in the next sections. Obviously, it is important to draw information from the fact that $\sigma:=\nabla^{\prime} \cdot m^{\prime}$ is controlled and that $\left|m^{\prime}\right|=1$. Following [5], we will do this by working with characteristic functions $\chi \in\{-1 / 2,1 / 2\}$ which have the property that the outer normal to the set $\{\chi=-1 / 2\}$ is 


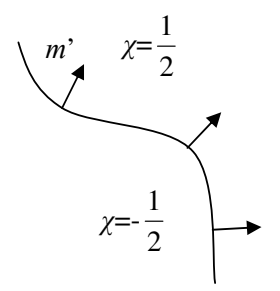

Fig. 9. The characteristic function $\chi$.

given by $m^{\prime}$ (see Figure 9). In the language of $B V$ functions, this means

$$
D^{\prime} \chi=m^{\prime}\left|D^{\prime} \chi\right|
$$

as an identity between measures. The purpose of 201 is that an integration by parts yields, for any localizing function $\eta$,

$$
\int \eta^{2} \chi \sigma d x^{\prime}=-\int \eta^{2}\left|D^{\prime} \chi\right|-\int \chi \nabla^{\prime} \eta^{2} \cdot m^{\prime} d x^{\prime} .
$$

The merit of 21 is that it gives a control of the length of the interface $\partial\{\chi=-1 / 2\}$ by $\sigma$ (and boundary data) (see Step 2 in the proof of Theorem 4). Let us be more precise: The energy $E_{\varepsilon}$ gives us a control over $\sigma$ in the form of

$$
\varepsilon \int \sigma^{2} d x^{\prime}
$$

and

$$
\int|h|^{2} d x=\left.\left.\frac{1}{2} \int|| \nabla^{\prime}\right|^{-1 / 2} \sigma\right|^{2} d x^{\prime}=\frac{1}{2} \int \frac{1}{\left|\xi^{\prime}\right|}|\mathcal{F}(\sigma)|^{2} d \xi^{\prime},
$$

where $\mathcal{F}(\sigma)$ denotes the Fourier transform of $\sigma$. The question which immediately arises is whether the control 233 of $\sigma$ is sufficient to estimate $\int \eta^{2} \chi \sigma d x^{\prime}$. We simplify the question even further: Are (23), $\int\left|D^{\prime} \chi\right|$ and sup $|\chi|$ enough to estimate the expression $\int \chi \sigma d x^{\prime}$ ? By duality, this question can be rephrased as follows: Can we control

$$
\left.\left.\int|| \nabla^{\prime}\right|^{1 / 2} \chi\right|^{2} d x^{\prime}=\int\left|\xi^{\prime}\right||\mathcal{F}(\chi)|^{2} d \xi^{\prime}
$$

by $\int\left|D^{\prime} \chi\right|$ and $\sup |\chi|$ ? The answer is no: For the 1-d characteristic function

$$
\chi= \begin{cases}1 / 2 & \text { for } x_{1}<0 \\ -1 / 2 & \text { for } x_{1}>0\end{cases}
$$

we have $|\mathcal{F}(\chi)|\left(\xi_{1}\right)=1 / \sqrt{2 \pi}\left|\xi_{1}\right|$ so that $\int\left|\xi_{1}\right||\mathcal{F}(\chi)|^{2} d \xi_{1}$ diverges logarithmically. However, the divergence is only logarithmic, which is also borne out by the fact that

$$
\left.\left.\int|| \nabla^{\prime}\right|^{1 / 2} \chi\right|^{2} d x^{\prime} \quad \text { and } \sup |\chi| \int\left|D^{\prime} \chi\right|
$$


have the same scaling. In fact, it follows from the analysis in [5] that

$$
\int_{\left\{1 \leq\left|\xi^{\prime}\right| \leq 1 / \varepsilon\right\}}\left|\xi^{\prime}\right||\mathcal{F}(\chi)|^{2} d \xi^{\prime} \leq \frac{2}{\pi}(|\ln \varepsilon|+C) \sup |\chi| \int\left|D^{\prime} \chi\right|
$$

for some universal constant $C$. (We will reprove a variant of 26 below.) Hence the Gagliardo-Nirenberg estimate in 25) holds when one cuts off the very small and very large length scales_-but the constant blows up logarithmically in the ratio of large and small cut-off length. Hence the control 22 is necessary to deal with the small length scales. It has to be combined with the estimate

$$
\int_{\left\{\left|\xi^{\prime}\right|>1 / \varepsilon\right\}}|\mathcal{F}(\chi)|^{2} d \xi^{\prime} \leq C \varepsilon \sup |\chi| \int\left|D^{\prime} \chi\right| .
$$

Finally, provided that

$$
\operatorname{supp} \chi \subset B_{1}^{\prime} \text {, }
$$

the large length scales are easily controlled,

$$
\int_{\left\{\left|\xi^{\prime}\right|<1\right\}}|\mathcal{F}(\chi)|^{2} d \xi^{\prime} \leq C(\sup |\chi|)^{2} .
$$

The combination of 23, 26, 27, and 28, yields

$$
\begin{aligned}
\left|\int \chi \sigma d x^{\prime}\right| \leq & \left(\frac{4}{\pi}(|\ln \varepsilon|+C) \sup |\chi| \int\left|D^{\prime} \chi\right| \int|h|^{2} d x\right)^{1 / 2} \\
& +C\left(\varepsilon \sup |\chi| \int\left|D^{\prime} \chi\right| \int \sigma^{2} d x^{\prime}\right)^{1 / 2}+C \sup |\chi|\left(\int|h|^{2} d x\right)^{1 / 2} \\
\leq & \left(\frac{4}{\pi}|\ln \varepsilon| \sup |\chi| \int\left|D^{\prime} \chi\right| \int|h|^{2} d x\right)^{1 / 2} \\
& +C\left(\varepsilon \int \sigma^{2} d x^{\prime}+\int|h|^{2} d x\right)^{1 / 2}\left(\sup |\chi|+\int\left|D^{\prime} \chi\right|\right) .
\end{aligned}
$$

In fact, we need the following estimate of the localized expression $\int \eta^{2} \chi \sigma d x^{\prime}$ :

Proposition 1. Let $h: \mathbb{R}^{3} \rightarrow \mathbb{R}^{3}$ and $\sigma: \mathbb{R}^{2} \rightarrow \mathbb{R}$ be related by

$$
\int_{\mathbb{R}^{3}} h \cdot \nabla \zeta d x=\int_{\mathbb{R}^{2}} \sigma \zeta d x^{\prime}, \quad \forall \zeta \in C_{c}^{\infty}\left(B_{1}\right),
$$

where $x^{\prime}=\left(x_{1}, x_{2}\right) \in \mathbb{R}^{2}$ and $x=\left(x^{\prime}, x_{3}\right) \in \mathbb{R}^{3}$. Let $\chi: \mathbb{R}^{2} \rightarrow \mathbb{R}$ be a bounded function of locally bounded variation and $\eta \in C_{c}^{\infty}\left(\mathbb{R}^{3}\right)$ be such that

$$
\operatorname{supp} \eta \subset B_{1} \subset \mathbb{R}^{3} \text {. }
$$


Then there exists a universal constant $C>0$ such that for all $\varepsilon \in(0,1]$,

$$
\begin{aligned}
\left|\int_{\mathbb{R}^{2}} \eta^{2} \chi \sigma d x^{\prime}\right| \leq & \left(\frac{4}{\pi}|\ln \varepsilon| \sup _{\mathbb{R}^{2}}|\chi| \int_{\mathbb{R}^{2}} \eta^{2}\left|D^{\prime} \chi\right| \int_{\mathbb{R}^{3}} \eta^{2}|h|^{2} d x\right)^{1 / 2} \\
& +C\left(\varepsilon \int_{B_{1}^{\prime}} \sigma^{2} d x^{\prime}+\int_{B_{1}}|h|^{2} d x\right)^{1 / 2} \\
& \times\left(\sup _{\mathbb{R}^{3}}|\eta|+\sup _{\mathbb{R}^{3}}|\nabla \eta|\right)\left(\sup _{\mathbb{R}^{3}}|\eta| \sup _{\mathbb{R}^{2}}|\chi|+\int_{\mathbb{R}^{2}}|\eta|\left|D^{\prime} \chi\right|\right),
\end{aligned}
$$

where $D^{\prime}$ denotes the in-plane derivatives $\left(\partial_{1}, \partial_{2}\right)$.

As a direct consequence, we have:

Corollary 1. Let $h: \mathbb{R}^{3} \rightarrow \mathbb{R}^{3}$ and $\sigma: \mathbb{R}^{2} \rightarrow \mathbb{R}$ be related by 30 . Let $\chi: \mathbb{R}^{2} \rightarrow \mathbb{R}$ be a bounded function of bounded variation such that supp $\chi \subset B_{1}^{\prime}$. Then there exists $a$ universal constant $C>0$ such that for all $\varepsilon \in(0,1]$,

$$
\begin{aligned}
\left|\int_{\mathbb{R}^{2}} \chi \sigma d x^{\prime}\right| \leq & \left(\frac{4}{\pi}|\ln \varepsilon| \sup _{\mathbb{R}^{2}}|\chi| \int_{B_{1}^{\prime}}\left|D^{\prime} \chi\right| \int_{B_{1}}|h|^{2} d x\right)^{1 / 2} \\
& +\frac{C}{d}\left(\varepsilon \int_{B_{1}^{\prime}} \sigma^{2} d x^{\prime}+\int_{B_{1}}|h|^{2} d x\right)^{1 / 2}\left(\sup _{\mathbb{R}^{2}}|\chi|+\int_{B_{1}^{\prime}}\left|D^{\prime} \chi\right|\right)
\end{aligned}
$$

where $d=\operatorname{dist}\left(\operatorname{supp} \chi, \partial B_{1}^{\prime}\right)>0$.

Proof of Proposition 1$]$ Let $C$ denote a generic universal constant. Our heuristic derivation of estimate 29] above was based on the decomposition in Fourier space, following [5]. However, Fourier space methods do not seem to be appropriate for our localized version. We thus need to find a real space counterpart of the Fourier space representation (24) and of the small scale cut-off $\left\{\left|\xi^{\prime}\right| \leq 1 / \varepsilon\right\}$ in (26). To circumvent [24), we work with the harmonic extension $\bar{\zeta}: \mathbb{R}^{3} \rightarrow \mathbb{R}$ of a function $\zeta: \mathbb{R}^{2} \rightarrow \mathbb{R}$, i.e.,

$$
\begin{cases}\Delta \bar{\zeta}=0 & \text { in } \mathbb{R}^{3} \backslash\left(\mathbb{R}^{2} \times\{0\}\right), \\ \bar{\zeta}(\cdot, 0)=\zeta & \text { on } \mathbb{R}^{2}\end{cases}
$$

Notice that equation 36 can be solved explicitly. The Fourier transform of 36 in the horizontal variables yields an ODE in $x_{3}$ with $\xi^{\prime}$ as a parameter. This ODE is solved by

$$
\mathcal{F}(\bar{\zeta})\left(\xi^{\prime}, x_{3}\right)=\mathcal{F}(\zeta)\left(\xi^{\prime}\right) e^{-\left|\xi^{\prime}\right|\left|x_{3}\right|}
$$

Therefore,

$$
\begin{aligned}
\int_{\mathbb{R}^{2}}\left|\xi^{\prime}\right||\mathcal{F}(\zeta)|^{2} d \xi^{\prime} & =\frac{1}{2} \int_{-\infty}^{\infty} \int_{\mathbb{R}^{2}}\left(\left|\xi^{\prime}\right|^{2}|\mathcal{F}(\bar{\zeta})|^{2}+\left|\partial_{3} \mathcal{F}(\bar{\zeta})\right|^{2}\right) d \xi^{\prime} d x_{3} \\
& =\frac{1}{2} \int_{\mathbb{R}^{3}}|\nabla \bar{\zeta}|^{2} d x
\end{aligned}
$$


To avoid the Fourier based decomposition into a small length scale part and the remainder, we introduce a convolution $\zeta_{\varepsilon}: \mathbb{R}^{2} \rightarrow \mathbb{R}$ of a function $\zeta: \mathbb{R}^{2} \rightarrow \mathbb{R}$ with a universal kernel $\rho_{\varepsilon}$ of the form

$$
\rho_{\varepsilon}\left(x^{\prime}\right)=\frac{1}{\varepsilon^{2}} \rho_{1}\left(\frac{x^{\prime}}{\varepsilon}\right), \quad \text { where } \quad \rho_{1} \in C_{c}^{\infty}\left(B_{1}^{\prime}\right) \text { is radial, } \rho_{1} \geq 0, \int_{B_{1}^{\prime}} \rho_{1}\left(x^{\prime}\right) d x^{\prime}=1 .
$$

Indeed, the convolution allows for a decomposition of $\zeta$ into the small scale part $\zeta-\zeta_{\varepsilon}$ and the remainder $\zeta_{\varepsilon}$.

We prove the estimate for $\chi \in W_{\text {loc }}^{1,1} \cap L^{\infty}\left(\mathbb{R}^{2}\right)$. In the general case of a function $\chi \in$ $B V_{\text {loc }} \cap L^{\infty}\left(\mathbb{R}^{2}\right)$, it will follow by a density argument, using a sequence $\left\{\chi_{\delta}\right\} \subset W_{\text {loc }}^{1,1} \cap$ $L^{\infty}\left(\mathbb{R}^{2}\right)$ such that $\chi_{\delta} \rightarrow \chi$ a.e. in $B_{1}^{\prime}, \sup _{\mathbb{R}^{2}}\left|\chi_{\delta}\right| \leq \sup _{\mathbb{R}^{2}}|\chi|$ and $\int_{B_{1}^{\prime}}\left|\nabla^{\prime} \chi_{\delta}\right| d x^{\prime} \rightarrow$ $\int_{B_{1}^{\prime}}\left|D^{\prime} \chi\right|$ (hence, $\left|D^{\prime} \chi_{\delta}\right| \rightarrow\left|D^{\prime} \chi\right|$ weak* as measures in $B_{1}^{\prime}$ ).

We rewrite the left-hand side of (32) as follows:

$$
\int_{\mathbb{R}^{2}} \eta^{2} \chi \sigma d x^{\prime}=\int_{\mathbb{R}^{2}} \eta \sigma\left(\eta \chi-(\eta \chi)_{\varepsilon}\right) d x^{\prime}+\int_{\mathbb{R}^{2}} \eta \sigma \overline{(\eta \chi)_{\varepsilon}} d x^{\prime},
$$

and by $(30)$ (where supp $\eta \overline{(\eta \chi)_{\varepsilon}} \subset B_{1}$ ),

$$
\begin{aligned}
\int_{\mathbb{R}^{2}} \eta \sigma \overline{(\eta \chi)_{\varepsilon}} d x^{\prime} & =\int_{\mathbb{R}^{3}} h \cdot \nabla\left(\eta \overline{(\eta \chi)_{\varepsilon}}\right) d x \\
& =\int_{\mathbb{R}^{3}} \overline{(\eta \chi)_{\varepsilon}} h \cdot \nabla \eta d x+\int_{\mathbb{R}^{3}} \eta h \cdot \nabla \overline{(\eta \chi)_{\varepsilon}} d x .
\end{aligned}
$$

Hence, we obtain the estimate

$$
\begin{aligned}
& \left|\int_{\mathbb{R}^{2}} \eta^{2} \chi \sigma d x^{\prime}\right| \leq\left(\int_{\mathbb{R}^{2}} \eta^{2} \sigma^{2} d x^{\prime}\right)^{1 / 2}\left(\int_{\mathbb{R}^{2}}\left|\eta \chi-(\eta \chi)_{\varepsilon}\right|^{2} d x^{\prime}\right)^{1 / 2} \\
& +\sup _{\mathbb{R}^{3}}\left|\overline{(\eta \chi)_{\varepsilon}}\right| \int_{\mathbb{R}^{3}}|h||\nabla \eta| d x \\
& +\left(\int_{\mathbb{R}^{3}} \eta^{2}|h|^{2} d x\right)^{1 / 2}\left(\int_{\mathbb{R}^{3}}\left|\nabla \overline{(\eta \chi)_{\varepsilon}}\right|^{2} d x\right)^{1 / 2} \\
& \stackrel{\text { 31 }}{\leq} \sup _{\mathbb{R}^{3}}|\eta|\left(\int_{B_{1}^{\prime}} \sigma^{2} d x^{\prime}\right)^{1 / 2}\left(\int_{\mathbb{R}^{2}}\left|\eta \chi-(\eta \chi)_{\varepsilon}\right|^{2} d x^{\prime}\right)^{1 / 2} \\
& +C \sup _{\mathbb{R}^{3}}|\nabla \eta| \sup _{\mathbb{R}^{3}}\left|\overline{(\eta \chi)_{\varepsilon}}\right|\left(\int_{B_{1}}|h|^{2} d x\right)^{1 / 2} \\
& +\left(\int_{\mathbb{R}^{3}} \eta^{2}|h|^{2} d x\right)^{1 / 2}\left(\int_{\mathbb{R}^{3}}\left|\nabla \overline{(\eta \chi)_{\varepsilon}}\right|^{2} d x\right)^{1 / 2} .
\end{aligned}
$$

As we shall see, only the term (40) contributes to the leading order term (32). We first address 38 and 39]. For 39, we observe that by the maximum principle,

$$
\sup _{\mathbb{R}^{3}}\left|\overline{(\eta \chi)_{\varepsilon}}\right| \leq \sup _{\mathbb{R}^{2}}\left|(\eta \chi)_{\varepsilon}\right| \leq \sup _{\mathbb{R}^{2}}|\eta \chi| \leq \sup _{\mathbb{R}^{3}}|\eta| \sup _{\mathbb{R}^{2}}|\chi|
$$


so that (39) can indeed be absorbed into (33). For (38), we have

$$
\begin{aligned}
\int_{\mathbb{R}^{2}}\left|\eta \chi-(\eta \chi)_{\varepsilon}\right|^{2} d x^{\prime} & \left.\leq \sup _{\mathbb{R}^{2}}\left|(\eta \chi)_{\varepsilon}\right|+\sup _{\mathbb{R}^{2}}|\eta \chi|\right) \int_{\mathbb{R}^{2}}\left|\eta \chi-(\eta \chi)_{\varepsilon}\right| d x^{\prime} \\
& \leq 2 \varepsilon \sup _{\mathbb{R}^{2}}|\eta \chi| \int_{\mathbb{R}^{2}}\left|\nabla^{\prime}(\eta \chi)\right| d x^{\prime} \\
& \leq 2 \varepsilon \sup _{\mathbb{R}^{2}}|\eta \chi| \int_{\mathbb{R}^{2}}\left(|\eta|\left|\nabla^{\prime} \chi\right|+|\chi|\left|\nabla^{\prime} \eta\right|\right) d x^{\prime} \\
& \leq C \varepsilon \sup _{\mathbb{R}^{3}}|\eta| \sup _{\mathbb{R}^{2}}|\chi|\left(\int_{\mathbb{R}^{2}}|\eta|\left|\nabla^{\prime} \chi\right| d x^{\prime}+\sup _{\mathbb{R}^{3}}|\nabla \eta| \sup _{\left.\mathbb{R}^{2}|\chi|\right) .}\right.
\end{aligned}
$$

Hence, 38 can be absorbed into 33.

We now turn to 40 . In order to have the desired inequality, it is sufficient to prove that

$$
\begin{aligned}
\int_{\mathbb{R}^{3}}\left|\nabla \overline{(\eta \chi)_{\varepsilon}}\right|^{2} d x \leq & \frac{4}{\pi}|\ln \varepsilon| \sup _{\mathbb{R}^{2}}|\chi| \int_{\mathbb{R}^{2}} \eta^{2}\left|\nabla^{\prime} \chi\right| d x^{\prime} \\
& +C\left(\sup _{\mathbb{R}^{3}}|\eta| \sup _{\mathbb{R}^{2}}|\chi|+\sup _{\mathbb{R}^{3}}|\nabla \eta| \sup _{\mathbb{R}^{2}}|\chi|+\int_{\mathbb{R}^{2}}|\eta|\left|\nabla^{\prime} \chi\right| d x^{\prime}\right)^{2} .
\end{aligned}
$$

We appeal to the following identity, which was already used in [5]:

$$
\int_{\mathbb{R}^{3}}|\nabla \bar{\phi}|^{2} d x=\frac{1}{2 \pi} \int_{\mathbb{R}^{2}} \frac{1}{\left|z^{\prime}\right|^{3}} \int_{\mathbb{R}^{2}}\left|\phi\left(x^{\prime}+z^{\prime}\right)-\phi\left(x^{\prime}\right)\right|^{2} d x^{\prime} d z^{\prime},
$$

which we apply to $\phi=(\eta \chi)_{\varepsilon}$. Actually, 43) is easy to establish. First of all, by homogeneity and isotropy, for every $\xi^{\prime} \in \mathbb{R}^{2}$,

$$
\begin{aligned}
\frac{1}{2 \pi} \int_{\mathbb{R}^{2}} \frac{1}{\left|z^{\prime}\right|^{3}} \mid 1-e^{\left.i \xi^{\prime} \cdot z^{\prime}\right|^{2} d z^{\prime}} & =\frac{\left|\xi^{\prime}\right|}{2 \pi} \int_{\mathbb{R}^{2}} \frac{1}{\left|\tilde{z}^{\prime}\right|^{3}}\left|1-e^{i \tilde{z}_{1}}\right|^{2} d \tilde{z}^{\prime} \\
& =\frac{2\left|\xi^{\prime}\right|}{\pi} \int_{0}^{2 \pi}\left(\int_{0}^{\infty} \frac{1}{r^{2}} \sin ^{2}\left(\frac{r|\cos \theta|}{2}\right) d r\right) d \theta \\
& =\frac{\left|\xi^{\prime}\right|}{\pi} \int_{0}^{2 \pi} \int_{0}^{\infty} \frac{|\cos \theta|}{s^{2}} \sin ^{2} s d s d \theta \\
& =\frac{\left|\xi^{\prime}\right|}{\pi} \int_{0}^{2 \pi}|\cos \theta| d \theta \int_{0}^{\infty} \frac{\sin ^{2} s}{s^{2}} d s=2\left|\xi^{\prime}\right| .
\end{aligned}
$$


(Here, we used the equalities $\int_{0}^{\infty} \frac{\sin ^{2} s}{s^{2}} d s=\int_{0}^{\infty} \frac{\sin 2 s}{s} d s=\frac{\pi}{2}$, see e.g. [1, 5.2.25].) By [37), we have

$$
\begin{aligned}
\int_{\mathbb{R}^{3}}|\nabla \bar{\phi}|^{2} d x & \stackrel{\text { 37] }}{=} 2 \int_{\mathbb{R}^{2}}\left|\xi^{\prime}\right|\left|\mathcal{F}(\phi)\left(\xi^{\prime}\right)\right|^{2} d \xi^{\prime} \\
& \stackrel{\text { 44 }}{=} \frac{1}{2 \pi} \int_{\mathbb{R}^{2}} \frac{1}{\left|z^{\prime}\right|^{3}} \int_{\mathbb{R}^{2}}\left|1-e^{i \xi^{\prime} \cdot z^{\prime}}\right|^{2}\left|\mathcal{F}(\phi)\left(\xi^{\prime}\right)\right|^{2} d \xi^{\prime} d z^{\prime} \\
& =\frac{1}{2 \pi} \int_{\mathbb{R}^{2}} \frac{1}{\left|z^{\prime}\right|^{3}} \int_{\mathbb{R}^{2}}\left|\phi\left(x^{\prime}+z^{\prime}\right)-\phi\left(x^{\prime}\right)\right|^{2} d x^{\prime} d z^{\prime},
\end{aligned}
$$

i.e., (43) holds. We split the $z^{\prime}$-integral on the right-hand side of (43) into three different regions:

$$
\begin{aligned}
& \frac{1}{2 \pi} \int_{\mathbb{R}^{2}} \frac{1}{\left|z^{\prime}\right|^{3}} \int_{\mathbb{R}^{2}}\left|(\eta \chi)_{\varepsilon}\left(x^{\prime}+z^{\prime}\right)-(\eta \chi)_{\varepsilon}\left(x^{\prime}\right)\right|^{2} d x^{\prime} d z^{\prime} \\
&=\frac{1}{2 \pi} \int_{\mathbb{R}^{2} \backslash B_{1}^{\prime}} \frac{1}{\left|z^{\prime}\right|^{3}} \int_{\mathbb{R}^{2}}\left|(\eta \chi)_{\varepsilon}\left(x^{\prime}+z^{\prime}\right)-(\eta \chi)_{\varepsilon}\left(x^{\prime}\right)\right|^{2} d x^{\prime} d z^{\prime} \\
&+\frac{1}{2 \pi} \int_{B_{\varepsilon}^{\prime}} \frac{1}{\left|z^{\prime}\right|^{3}} \int_{\mathbb{R}^{2}}\left|(\eta \chi)_{\varepsilon}\left(x^{\prime}+z^{\prime}\right)-(\eta \chi)_{\varepsilon}\left(x^{\prime}\right)\right|^{2} d x^{\prime} d z^{\prime} \\
&+\frac{1}{2 \pi} \int_{B_{1}^{\prime} \backslash B_{\varepsilon}^{\prime}} \frac{1}{\left|z^{\prime}\right|^{3}} \int_{\mathbb{R}^{2}}\left|(\eta \chi)_{\varepsilon}\left(x^{\prime}+z^{\prime}\right)-(\eta \chi)_{\varepsilon}\left(x^{\prime}\right)\right|^{2} d x^{\prime} d z^{\prime} .
\end{aligned}
$$

As we shall see, only the intermediate term (47) contributes to the leading order term 411.

We first address 45 and 46 . We start with the term 45) corresponding to the long wave length (i.e., $\left|z^{\prime}\right| \geq 1$ ). Since

$$
\begin{aligned}
\int_{\mathbb{R}^{2}}\left|(\eta \chi)_{\varepsilon}\left(x^{\prime}+z^{\prime}\right)-(\eta \chi)_{\varepsilon}\left(x^{\prime}\right)\right|^{2} d x^{\prime} & \leq 2 \int_{\mathbb{R}^{2}}\left|(\eta \chi)_{\varepsilon}\right|^{2} d x^{\prime} \\
& \leq 2 \int_{\mathbb{R}^{2}}|\eta \chi|^{2} d x^{\prime} \stackrel{\text { 31| }}{\leq} \sup _{\mathbb{R}^{3}}|\eta|^{2} \sup _{\mathbb{R}^{2}}|\chi|^{2},
\end{aligned}
$$

we obtain

$$
\begin{aligned}
\frac{1}{2 \pi} \int_{\mathbb{R}^{2} \backslash B_{1}^{\prime}} \frac{1}{\left|z^{\prime}\right|^{3}} & \int_{\mathbb{R}^{2}}\left|(\eta \chi)_{\varepsilon}\left(x^{\prime}+z^{\prime}\right)-(\eta \chi)_{\varepsilon}\left(x^{\prime}\right)\right|^{2} d x^{\prime} d z^{\prime} \\
& \leq C \sup _{\mathbb{R}^{3}}|\eta|^{2} \sup _{\mathbb{R}^{2}}|\chi|^{2} \int_{\mathbb{R}^{2} \backslash B_{1}^{\prime}} \frac{1}{\left|z^{\prime}\right|^{3}} d z^{\prime} \leq C \sup _{\mathbb{R}^{3}}|\eta|^{2} \sup _{\mathbb{R}^{2}}|\chi|^{2},
\end{aligned}
$$

i.e., 45) is absorbed by (42). We now tackle the short wave length term 446. We have

$$
\begin{aligned}
\int_{\mathbb{R}^{2}}\left|(\eta \chi)_{\varepsilon}\left(x^{\prime}+z^{\prime}\right)-(\eta \chi)_{\varepsilon}\left(x^{\prime}\right)\right|^{2} d x^{\prime} \leq\left|z^{\prime}\right|^{2} \int_{\mathbb{R}^{2}}\left|\nabla^{\prime}(\eta \chi)_{\varepsilon}\right|^{2} d x^{\prime} \\
\leq\left|z^{\prime}\right|^{2} \sup _{\mathbb{R}^{2}}\left|\nabla^{\prime}(\eta \chi)_{\varepsilon}\right| \int_{\mathbb{R}^{2}}\left|\nabla^{\prime}(\eta \chi)_{\varepsilon}\right| d x^{\prime} \leq \frac{C}{\varepsilon}\left|z^{\prime}\right|^{2} \sup _{\mathbb{R}^{2}}|\eta \chi| \int_{\mathbb{R}^{2}}\left|\nabla^{\prime}(\eta \chi)\right| d x^{\prime}
\end{aligned}
$$


and thus,

$$
\begin{aligned}
& \frac{1}{2 \pi} \int_{B_{\varepsilon}^{\prime}} \frac{1}{\left|z^{\prime}\right|^{3}} \int_{\mathbb{R}^{2}}\left|(\eta \chi)_{\varepsilon}\left(x^{\prime}+z^{\prime}\right)-(\eta \chi)_{\varepsilon}\left(x^{\prime}\right)\right|^{2} d x^{\prime} d z^{\prime} \\
& \stackrel{\text { 31 }}{\leq} C \sup _{\mathbb{R}^{3}}|\eta| \sup _{\mathbb{R}^{2}}|\chi|\left(\sup _{\mathbb{R}^{3}}|\nabla \eta| \sup _{\mathbb{R}^{2}}|\chi|+\int_{\mathbb{R}^{2}}|\eta|\left|\nabla^{\prime} \chi\right| d x^{\prime}\right) \frac{1}{\varepsilon} \int_{B_{\varepsilon}^{\prime}} \frac{d z^{\prime}}{\left|z^{\prime}\right|},
\end{aligned}
$$

i.e., (46) can also be absorbed by 42].

We finally address the medium wave length term 477 . We start by observing that

$$
\int_{\mathbb{R}^{2}}\left|(\eta \chi)_{\varepsilon}\left(x^{\prime}+z^{\prime}\right)-(\eta \chi)_{\varepsilon}\left(x^{\prime}\right)\right|^{2} d x^{\prime} \leq \int_{\mathbb{R}^{2}}\left|(\eta \chi)\left(x^{\prime}+z^{\prime}\right)-(\eta \chi)\left(x^{\prime}\right)\right|^{2} d x^{\prime} .
$$

We rewrite the integrand as

$$
\begin{aligned}
\left|(\eta \chi)\left(x^{\prime}+z^{\prime}\right)-(\eta \chi)\left(x^{\prime}\right)\right|^{2}= & \left(\chi\left(x^{\prime}+z^{\prime}\right)-\chi\left(x^{\prime}\right)\right) \int_{0}^{1} \eta^{2}\left(x^{\prime}+t z^{\prime}\right) \nabla^{\prime} \chi\left(x^{\prime}+t z^{\prime}\right) \cdot z^{\prime} d t \\
& + \text { remainder. }
\end{aligned}
$$

To do so, we proceed as follows:

$$
\begin{aligned}
& (\eta \chi)\left(x^{\prime}+z^{\prime}\right)-(\eta \chi)\left(x^{\prime}\right)=\int_{0}^{1} \nabla^{\prime}(\eta \chi)\left(x^{\prime}+t z^{\prime}\right) \cdot z^{\prime} d t \\
& \quad=\int_{0}^{1} \eta\left(x^{\prime}+t z^{\prime}\right) \nabla^{\prime} \chi\left(x^{\prime}+t z^{\prime}\right) \cdot z^{\prime} d t+\int_{0}^{1} \chi\left(x^{\prime}+t z^{\prime}\right) \nabla^{\prime} \eta\left(x^{\prime}+t z^{\prime}\right) \cdot z^{\prime} d t
\end{aligned}
$$

and thus,

$$
\begin{aligned}
& \left|(\eta \chi)\left(x^{\prime}+z^{\prime}\right)-(\eta \chi)\left(x^{\prime}\right)\right|^{2} \\
& =\left(\chi\left(x^{\prime}+z^{\prime}\right)-\chi\left(x^{\prime}\right)\right) \int_{0}^{1} \eta^{2}\left(x^{\prime}+t z^{\prime}\right) \nabla^{\prime} \chi\left(x^{\prime}+t z^{\prime}\right) \cdot z^{\prime} d t \\
& \quad+\chi\left(x^{\prime}+z^{\prime}\right) \int_{0}^{1}\left(\eta\left(x^{\prime}+z^{\prime}\right)-\eta\left(x^{\prime}+t z^{\prime}\right)\right) \eta\left(x^{\prime}+t z^{\prime}\right) \nabla^{\prime} \chi\left(x^{\prime}+t z^{\prime}\right) \cdot z^{\prime} d t \\
& \quad-\chi\left(x^{\prime}\right) \int_{0}^{1}\left(\eta\left(x^{\prime}\right)-\eta\left(x^{\prime}+t z^{\prime}\right)\right) \eta\left(x^{\prime}+t z^{\prime}\right) \nabla^{\prime} \chi\left(x^{\prime}+t z^{\prime}\right) \cdot z^{\prime} d t \\
& \quad+\int_{0}^{1} \eta\left(x^{\prime}+t z^{\prime}\right) \nabla^{\prime} \chi\left(x^{\prime}+t z^{\prime}\right) \cdot z^{\prime} d t \int_{0}^{1} \chi\left(x^{\prime}+t z^{\prime}\right) \nabla^{\prime} \eta\left(x^{\prime}+t z^{\prime}\right) \cdot z^{\prime} d t \\
& \quad+\left(\int_{0}^{1} \chi\left(x^{\prime}+t z^{\prime}\right) \nabla^{\prime} \eta\left(x^{\prime}+t z^{\prime}\right) \cdot z^{\prime} d t\right)^{2} .
\end{aligned}
$$


This yields the estimate

$$
\begin{aligned}
\left|(\eta \chi)\left(x^{\prime}+z^{\prime}\right)-(\eta \chi)\left(x^{\prime}\right)\right|^{2} \leq & 2 \sup _{\mathbb{R}^{2}}|\chi| \int_{0}^{1} \eta^{2}\left(x^{\prime}+t z^{\prime}\right)\left|\nabla^{\prime} \chi\left(x^{\prime}+t z^{\prime}\right) \cdot z^{\prime}\right| d t \\
& +3\left|z^{\prime}\right|^{2} \sup _{\mathbb{R}^{2}}|\chi| \sup _{\mathbb{R}^{3}}|\nabla \eta| \int_{0}^{1}\left|\eta\left(x^{\prime}+t z^{\prime}\right)\right|\left|\nabla^{\prime} \chi\left(x^{\prime}+t z^{\prime}\right)\right| d t \\
& +\left|z^{\prime}\right|^{2} \sup _{\mathbb{R}^{2}}|\chi|^{2} \sup _{\mathbb{R}^{3}}|\nabla \eta| \int_{0}^{1}\left|\nabla^{\prime} \eta\left(x^{\prime}+t z^{\prime}\right)\right| d t .
\end{aligned}
$$

Integration in $x^{\prime}$ gives

$$
\begin{aligned}
& \int_{\mathbb{R}^{2}}\left|(\eta \chi)\left(x^{\prime}+z^{\prime}\right)-(\eta \chi)\left(x^{\prime}\right)\right|^{2} d x^{\prime} \\
& \leq 2 \sup _{\mathbb{R}^{2}}|\chi| \int_{\mathbb{R}^{2}} \eta^{2}\left|\nabla^{\prime} \chi \cdot z^{\prime}\right| d x^{\prime}+3\left|z^{\prime}\right|^{2} \sup _{\mathbb{R}^{2}}|\chi| \sup _{\mathbb{R}^{3}}|\nabla \eta| \int_{\mathbb{R}^{2}}|\eta|\left|\nabla^{\prime} \chi\right| d x^{\prime} \\
&+\left|z^{\prime}\right|^{2} \sup _{\mathbb{R}^{2}}|\chi|^{2} \sup _{\mathbb{R}^{3}}|\nabla \eta| \int_{\mathbb{R}^{2}}\left|\nabla^{\prime} \eta\right| d x^{\prime} \\
& \leq 2 \sup _{\mathbb{R}^{2}}|\chi| \int_{\mathbb{R}^{2}} \eta^{2}\left|\nabla^{\prime} \chi \cdot z^{\prime}\right| d x^{\prime} \\
&+C\left|z^{\prime}\right|^{2} \sup _{\mathbb{R}^{3}}|\nabla \eta| \sup _{\mathbb{R}^{2}}|\chi|\left(\sup _{\mathbb{R}^{3}}|\nabla \eta| \sup _{\mathbb{R}^{2}}|\chi|+\int_{\mathbb{R}^{2}}|\eta|\left|\nabla^{\prime} \chi\right| d x^{\prime}\right) .
\end{aligned}
$$

Integration in $z^{\prime}$ yields

$$
\begin{aligned}
& \int_{B_{1}^{\prime} \backslash B_{\varepsilon}^{\prime}} \frac{1}{\left|z^{\prime}\right|^{3}} \int_{\mathbb{R}^{2}}\left|(\eta \chi)\left(x^{\prime}+z^{\prime}\right)-(\eta \chi)\left(x^{\prime}\right)\right|^{2} d x^{\prime} d z^{\prime} \\
& \leq 2 \sup _{\mathbb{R}^{2}}|\chi| \int_{\mathbb{R}^{2}} \eta^{2}\left(x^{\prime}\right) \int_{B_{1}^{\prime} \backslash B_{\varepsilon}^{\prime}} \frac{1}{\left|z^{\prime}\right|^{3}}\left|\nabla^{\prime} \chi\left(x^{\prime}\right) \cdot z^{\prime}\right| d z^{\prime} d x^{\prime} \\
& \quad+C\left(\sup _{\mathbb{R}^{3}}|\eta| \sup _{\mathbb{R}^{2}}|\chi|+\sup _{\mathbb{R}^{3}}|\nabla \eta| \sup _{\mathbb{R}^{2}}|\chi|+\int_{\mathbb{R}^{2}}|\eta|\left|\nabla^{\prime} \chi\right| d x^{\prime}\right)^{2} \int_{B_{1}^{\prime} \backslash B_{\varepsilon}^{\prime}} \frac{d z^{\prime}}{\left|z^{\prime}\right|} .
\end{aligned}
$$

Notice that for any $v^{\prime} \in \mathbb{R}^{2}$,

$$
\begin{aligned}
\int_{B_{1}^{\prime} \backslash B_{\varepsilon}^{\prime}} \frac{1}{\left|z^{\prime}\right|^{3}}\left|v^{\prime} \cdot z^{\prime}\right| d z^{\prime} & =\int_{0}^{2 \pi} \int_{\varepsilon}^{1} \frac{1}{r^{3}}\left|v^{\prime} \cdot\left(\begin{array}{c}
r \cos \theta \\
r \sin \theta
\end{array}\right)\right| r d r d \theta \\
& =\left|v^{\prime}\right| \int_{0}^{2 \pi}|\cos \theta| d \theta \int_{\varepsilon}^{1} \frac{1}{r} d r=4|\ln \varepsilon|\left|v^{\prime}\right| .
\end{aligned}
$$


Hence (50) turns into

$$
\begin{aligned}
\frac{1}{2 \pi} \int_{B_{1}^{\prime} \backslash B_{\varepsilon}^{\prime}} \frac{1}{\left|z^{\prime}\right|^{3}} \int_{\mathbb{R}^{2}}\left|(\eta \chi)\left(x^{\prime}+z^{\prime}\right)-(\eta \chi)\left(x^{\prime}\right)\right|^{2} d x^{\prime} d z^{\prime} \leq \frac{4}{\pi}|\ln \varepsilon| \sup _{\mathbb{R}^{2}}|\chi| \int_{\mathbb{R}^{2}} \eta^{2}\left|\nabla^{\prime} \chi\right| d x^{\prime} \\
+C\left(\sup _{\mathbb{R}^{3}}|\eta| \sup _{\mathbb{R}^{2}}|\chi|+\sup _{\mathbb{R}^{3}}|\nabla \eta| \sup _{\mathbb{R}^{2}}|\chi|+\int_{\mathbb{R}^{2}}|\eta|\left|\nabla^{\prime} \chi\right| d x^{\prime}\right)^{2}
\end{aligned}
$$

Combining identity (43) with the estimates (48), (49) and (51), we conclude that inequality $41, \& 42$ holds.

Proof of Corollary 1. It directly follows from Proposition 1 by choosing $\eta \in C_{c}^{\infty}\left(B_{1}\right)$ such that

$$
\eta=1 \quad \text { on supp } \chi, \quad|\eta| \leq 1 \quad \text { and } \quad|\nabla \eta| \leq C / d \quad \text { in } B_{1} .
$$

By rescaling length in Proposition 1 from unity to some $R>0$, we obtain:

Corollary 2. Let $R>0$ and $x_{0}=\left(x_{0}^{\prime}, 0\right) \in \mathbb{R}^{2} \times\{0\}$. Let $h: \mathbb{R}^{3} \rightarrow \mathbb{R}^{3}$ and $\sigma: \mathbb{R}^{2} \rightarrow \mathbb{R}$ be related by

$$
\int_{\mathbb{R}^{3}} h \cdot \nabla \zeta d x=\int_{\mathbb{R}^{2}} \sigma \zeta d x^{\prime}, \quad \forall \zeta \in C_{c}^{\infty}\left(B\left(x_{0}, R\right)\right) .
$$

Let $\chi: \mathbb{R}^{2} \rightarrow \mathbb{R}$ be a bounded function of locally bounded variation and $\eta \in C_{c}^{\infty}\left(\mathbb{R}^{3}\right)$ be such that

$$
\operatorname{supp} \eta \subset B\left(x_{0}, R\right) \subset \mathbb{R}^{3} .
$$

Then there exists a universal constant $C>0$ such that for all $\varepsilon \in(0, R]$,

$$
\begin{aligned}
\left|\int_{\mathbb{R}^{2}} \eta^{2} \chi \sigma d x^{\prime}\right| \leq & \left(\frac{4}{\pi}|\ln \varepsilon| \sup _{\mathbb{R}^{2}}|\chi| \int_{\mathbb{R}^{2}} \eta^{2}\left|D^{\prime} \chi\right| \int_{\mathbb{R}^{3}} \eta^{2}|h|^{2} d x\right)^{1 / 2} \\
& +C\left(1+|\ln R|^{1 / 2}\right)\left(\varepsilon \int_{B^{\prime}\left(x_{0}^{\prime}, R\right)} \sigma^{2} d x^{\prime}+\int_{B\left(x_{0}, R\right)}|h|^{2} d x\right)^{1 / 2} \\
\times & \left.\sup _{\mathbb{R}^{3}}|\eta|+R \sup _{\mathbb{R}^{3}}|\nabla \eta|\right)\left(R^{1 / 2} \sup _{\mathbb{R}^{3}}|\eta| \sup _{\mathbb{R}^{2}}|\chi|+R^{-1 / 2} \int_{\mathbb{R}^{2}}|\eta|\left|D^{\prime} \chi\right|\right) .
\end{aligned}
$$

Proof. The change of variables $x=R \hat{x}+x_{0}$ (and $\varepsilon=R \hat{\varepsilon}$ ) preserves (30) and turns (52) into supp $\eta \subset \hat{B}_{1}$, so that we may apply Proposition 1 . It yields, in the original variables,

$$
\begin{aligned}
\left|R^{-2} \int_{\mathbb{R}^{2}} \eta^{2} \chi \sigma d x^{\prime}\right| \leq & \left(\frac{4}{\pi}\left|\ln \frac{\varepsilon}{R}\right| \sup _{\mathbb{R}^{2}}|\chi| R^{-1} \int_{\mathbb{R}^{2}} \eta^{2}\left|D^{\prime} \chi\right| R^{-3} \int_{\mathbb{R}^{3}} \eta^{2}|h|^{2} d x\right)^{1 / 2} \\
& +C\left(\frac{\varepsilon}{R} R^{-2} \int_{B^{\prime}\left(x_{0}^{\prime}, R\right)} \sigma^{2} d x^{\prime}+R^{-3} \int_{B\left(x_{0}, R\right)}|h|^{2} d x\right)^{1 / 2} \\
& \times\left(\sup _{\mathbb{R}^{3}}|\eta|+R \sup _{\mathbb{R}^{3}}|\nabla \eta|\right)\left(\sup _{\mathbb{R}^{3}}|\eta| \sup _{\mathbb{R}^{2}}|\chi|+R^{-1} \int_{\mathbb{R}^{2}}|\eta|\left|D^{\prime} \chi\right|\right),
\end{aligned}
$$


that is,

$$
\begin{aligned}
\left|\int_{\mathbb{R}^{2}} \eta^{2} \chi \sigma d x^{\prime}\right| \leq & \left(\frac{4}{\pi}(|\ln \varepsilon|+|\ln R|) \sup _{\mathbb{R}^{2}}|\chi| \int_{\mathbb{R}^{2}} \eta^{2}\left|D^{\prime} \chi\right| \int_{\mathbb{R}^{3}} \eta^{2}|h|^{2} d x\right)^{1 / 2} \\
& +C\left(\varepsilon \int_{B^{\prime}\left(x_{0}^{\prime}, R\right)} \sigma^{2} d x^{\prime}+\int_{B\left(x_{0}, R\right)}|h|^{2} d x\right)^{1 / 2} \\
& \left.\times \sup _{\mathbb{R}^{3}}|\eta|+R \sup _{\mathbb{R}^{3}}|\nabla \eta|\right)\left(R^{1 / 2} \sup _{\mathbb{R}^{3}}|\eta| \sup _{\mathbb{R}^{2}}|\chi|+R^{-1 / 2} \int_{\mathbb{R}^{2}}|\eta|\left|D^{\prime} \chi\right|\right) .
\end{aligned}
$$

The conclusion is now straightforward.

If one drops the test function $\eta$ and localizes the function $\chi$ in Corollary 2 , the following result comes out:

Corollary 3. Let $R>0$ and $x_{0}=\left(x_{0}^{\prime}, 0\right) \in \mathbb{R}^{2} \times\{0\}$. Let $h: \mathbb{R}^{3} \rightarrow \mathbb{R}^{3}$ and $\sigma: \mathbb{R}^{2} \rightarrow \mathbb{R}$ be related by

$$
\int_{\mathbb{R}^{3}} h \cdot \nabla \zeta d x=\int_{\mathbb{R}^{2}} \sigma \zeta d x^{\prime}, \quad \forall \zeta \in C_{c}^{\infty}\left(B\left(x_{0}, 2 R\right)\right) .
$$

Let $\chi: \mathbb{R}^{2} \rightarrow \mathbb{R}$ be a bounded function of bounded variation such that $\operatorname{supp} \chi \subseteq$ $\bar{B}^{\prime}\left(x_{0}^{\prime}, R\right) \subset \mathbb{R}^{2}$. Then there exists a constant $C(R)>0$ only depending on $R$ such that for all $\varepsilon \in(0,2 R]$,

$$
\begin{aligned}
\left|\int_{\mathbb{R}^{2}} \chi \sigma d x^{\prime}\right| & \leq\left(\frac{4}{\pi}|\ln \varepsilon| \sup _{\mathbb{R}^{2}}|\chi| \int_{\mathbb{R}^{2}}\left|D^{\prime} \chi\right| \int_{B\left(x_{0}, 2 R\right)}|h|^{2} d x\right)^{1 / 2} \\
& +C(R)\left(\varepsilon \int_{B^{\prime}\left(x_{0}^{\prime}, 2 R\right)} \sigma^{2} d x^{\prime}+\int_{B\left(x_{0}, 2 R\right)}|h|^{2} d x\right)^{1 / 2}\left(\sup _{\mathbb{R}^{2}}|\chi|+\int_{\mathbb{R}^{2}}\left|D^{\prime} \chi\right|\right) .
\end{aligned}
$$

Proof. Let $\eta \in C_{c}^{\infty}\left(B\left(x_{0}, 2 R\right)\right)$ be such that

$$
\eta=1 \quad \text { in } B^{\prime}\left(x_{0}^{\prime}, R\right) \times\{0\}, \quad|\eta| \leq 1 \quad \text { and } \quad|\nabla \eta| \leq C / R \quad \text { in } B\left(x_{0}, 2 R\right),
$$

where $C>0$ is some generic constant. We apply Corollary 2

$$
\begin{aligned}
\left|\int_{\mathbb{R}^{2}} \eta^{2} \chi \sigma d x^{\prime}\right| \stackrel{54]}{\leq} & \left(\frac{4}{\pi}|\ln \varepsilon| \sup _{\mathbb{R}^{2}}|\chi| \int_{\mathbb{R}^{2}}\left|D^{\prime} \chi\right| \int_{B\left(x_{0}, 2 R\right)}|h|^{2} d x\right)^{1 / 2} \\
& +C\left(1+|\ln R|^{1 / 2}\right)\left(\varepsilon \int_{B^{\prime}\left(x_{0}^{\prime}, 2 R\right)} \sigma^{2} d x^{\prime}+\int_{B\left(x_{0}, 2 R\right)}|h|^{2} d x\right)^{1 / 2} \\
& \times\left(R^{1 / 2} \sup _{\mathbb{R}^{2}}|\chi|+R^{-1 / 2} \int_{\mathbb{R}^{2}}\left|D^{\prime} \chi\right|\right),
\end{aligned}
$$

and the conclusion is straightforward.

A periodic version of Proposition 1 is the following: 
Corollary 4. Let $L>0$. Let $h: \mathbb{R}^{3} \rightarrow \mathbb{R}^{3}$ and $\sigma: \mathbb{R}^{2} \rightarrow \mathbb{R}$ be related by

$$
\int_{\mathbb{R}^{3}} h \cdot \nabla \zeta d x=\int_{\mathbb{R}^{2}} \sigma \zeta d x^{\prime}, \quad \forall \zeta \in C_{c}^{\infty}\left(\mathbb{R}^{3}\right) .
$$

Let $\chi: \mathbb{R}^{2} \rightarrow \mathbb{R}$ be a bounded function of bounded variation in $\mathbb{R} \times[0, L)$ and $\eta \in$ $C^{\infty}\left(\mathbb{R}^{3}\right)$ be such that

$$
\operatorname{supp} \eta \subset(-2,2) \times \mathbb{R} \times(-1,1) .
$$

Assume that

$$
h, \sigma, \chi \text { and } \eta \text { are L-periodic in } x_{2} \text {. }
$$

Then there exists a constant $C(L)>0$ only depending on $L$ such that for all $\varepsilon \in(0, L]$,

$$
\begin{aligned}
\left|\int_{\mathbb{R} \times[0, L)} \eta^{2} \chi \sigma d x^{\prime}\right| \leq & \left(\frac{4}{\pi}|\ln \varepsilon| \sup _{\mathbb{R}^{2}}|\chi| \int_{\mathbb{R} \times[0, L)} \eta^{2}\left|D^{\prime} \chi\right| \int_{\mathbb{R} \times[0, L) \times \mathbb{R}} \eta^{2}|h|^{2} d x\right)^{1 / 2} \\
& +C(L)\left(\varepsilon \int_{\mathbb{R} \times[0, L)} \sigma^{2} d x^{\prime}+\int_{\mathbb{R} \times[0, L) \times \mathbb{R}}|h|^{2} d x\right)^{1 / 2} \\
\times & \left.\sup _{\mathbb{R}^{3}}|\eta|+\sup _{\mathbb{R}^{3}}|\nabla \eta|\right)\left(\sup _{\left.\mathbb{R}^{3}|\eta| \sup _{\mathbb{R}^{2}}|\chi|+\int_{\mathbb{R} \times[0, L)}|\eta|\left|D^{\prime} \chi\right|\right) .}\right.
\end{aligned}
$$

Proof. Select a universal $\zeta \in C_{c}^{\infty}(\mathbb{R})$ such that

$$
\operatorname{supp} \zeta \subset(-1,1), \quad|\zeta| \leq 1, \quad \sum_{k \in \mathbb{Z}} \zeta^{2}\left(x_{2}+k\right)=1, \quad \forall x_{2} \in \mathbb{R}
$$

and set

$$
\tilde{\eta}\left(x_{1}, x_{2}, x_{3}\right)=\zeta\left(x_{2} / L\right) \eta\left(x_{1}, x_{2}, x_{3}\right), \quad \forall\left(x_{1}, x_{2}, x_{3}\right) \in \mathbb{R}^{3} .
$$

In view of (55) and (58) we have supp $\tilde{\eta} \subset B_{R}$ for some radius

$$
\tilde{L} \leq R \leq 2 \tilde{L}
$$

where $\tilde{L}=\max \{2, L\}$. Hence, we may apply 53 to $\sigma, h, \chi$ and $\tilde{\eta}$. Notice that because of 56) and 58),

$$
\begin{aligned}
\int_{\mathbb{R}^{2}} \tilde{\eta}^{2} \chi \sigma d x^{\prime} & =\int_{\mathbb{R} \times[0, L)} \eta^{2} \chi \sigma d x^{\prime}, & & \int_{\mathbb{R}^{2}} \tilde{\eta}^{2}\left|D^{\prime} \chi\right|=\int_{\mathbb{R} \times[0, L)} \eta^{2}\left|D^{\prime} \chi\right|, \\
\int_{\mathbb{R}^{3}} \tilde{\eta}^{2}|h|^{2} d x & =\int_{\mathbb{R} \times[0, L) \times \mathbb{R}} \eta^{2}|h|^{2} d x, & & \int_{\mathbb{R}^{2}}|\tilde{\eta}|\left|D^{\prime} \chi\right| \leq C \int_{\mathbb{R} \times[0, L)}|\eta|\left|D^{\prime} \chi\right| .
\end{aligned}
$$

Furthermore, by (56) and 60) we have

$$
\int_{B_{R}^{\prime}} \sigma^{2} d x^{\prime} \leq C \frac{\tilde{L}}{L} \int_{\mathbb{R} \times[0, L)} \sigma^{2} d x^{\prime}, \quad \int_{B_{R}}|h|^{2} d x \leq C \frac{\tilde{L}}{L} \int_{\mathbb{R} \times[0, L) \times \mathbb{R}}|h|^{2} d x .
$$

Finally, it follows from 58 and 59 that

$$
\sup _{\mathbb{R}^{3}}|\tilde{\eta}| \leq \sup _{\mathbb{R}^{3}}|\eta|, \quad \sup _{\mathbb{R}^{3}}|\nabla \tilde{\eta}| \leq \frac{C}{L} \sup _{\mathbb{R}^{3}}|\eta|+\sup _{\mathbb{R}^{3}}|\nabla \eta| .
$$

Hence, 53 yields 57. 
Remark 3. The conclusion of Corollary 4 holds true for a more general support of $\eta$ than (55) (for example, $(-a, a) \times \mathbb{R} \times(a, a)$ for every $a>0)$. The choice of the interval $(-2,2)$ in (55) (as support in $x_{1}$ variable) is needed in the proof of Theorem 1 due to the choice of the boundary data (1).

\section{Compactness of the Néel wall}

This section is devoted to the proof of the compactness result for magnetizations in the energy regime $O(1 /|\ln \varepsilon|)$ :

Proof of Theorem 4 We proceed in several steps:

Step 1. Some preliminaries. Since $\left|m_{k}^{\prime}\right|=1$ in $B_{1}^{\prime}$, it follows that the sequence $\left\{\left\|m_{k}^{\prime}\right\|_{L^{\infty}\left(B_{1}^{\prime}\right)}\right\}_{k \in \mathbb{N}}$ is bounded, and therefore there exists $m^{\prime} \in L^{\infty}\left(B_{1}^{\prime}, \mathbb{R}^{2}\right)$ such that up to a subsequence,

$$
m_{k}^{\prime} \rightarrow m^{\prime} \quad \text { weak }^{*} \text { in } L^{\infty}
$$

In particular,

$$
\left|m^{\prime}\right| \leq 1 \quad \text { a.e. in } B_{1}^{\prime} .
$$

In order to have strong convergence in some $L^{p}$ with $1 \leq p<\infty$, we need to show that $\left|m^{\prime}\right|=1$ a.e. in $B_{1}^{\prime}$. Indeed, that will imply $\left\|m_{k}^{\prime}\right\|_{L^{2}\left(B_{1}^{\prime}\right)} \rightarrow\left\|m^{\prime}\right\|_{L^{2}\left(B_{1}^{\prime}\right)}$ and by the weak convergence in $L^{2}$, it will lead to the strong convergence in $L^{2}$, and then in any other $L^{p}$, $1 \leq p<\infty$.

We introduce the finite positive measures $\left\{e_{k}\right\}_{k \in \mathbb{N}} \subset \mathcal{M}\left(B_{1}\right)$ as

$$
\int_{\mathbb{R}^{3}} \zeta d e_{k}=\frac{2}{\pi}\left|\ln \varepsilon_{k}\right|\left(\varepsilon_{k} \int_{\mathbb{R}^{2}} \zeta\left|\nabla^{\prime} \cdot m_{k}^{\prime}\right|^{2} d x^{\prime}+\int_{\mathbb{R}^{3}} \zeta\left|h_{k}\right|^{2} d x\right), \quad \forall \zeta \in C_{c}\left(B_{1}\right)
$$

Then by (17), the family $\left\{e_{k}\right\}_{k \in \mathbb{N}}$ of positive measures is bounded in $\mathcal{M}\left(B_{1}\right)$, and hence there exists a positive measure $e \in \mathcal{M}\left(B_{1}\right)$ such that

$$
e_{k} \rightarrow e \quad \text { weak }^{*} \text { in } \mathcal{M}\left(B_{1}\right)
$$

for a subsequence.

Step 2. Some topology. Let $x_{0}^{\prime} \in B_{1}^{\prime}$. Using the technique of [5], we will identify the "characteristic" of $m^{\prime}$ passing through $x_{0}^{\prime}$. Recall that the admissible magnetizations $m_{k}^{\prime}$ are assumed to be smooth and consider the autonomous equation

$$
\dot{X}=m_{k}^{\prime \perp}(X) \text {. }
$$

First of all, 65) has no critical point and no cycle (i.e., closed orbit): Since $\left|m_{k}^{\prime}{ }^{\perp}\right|=1$ in $B_{1}^{\prime}$ and $m_{k}^{\prime}{ }^{\perp}$ is smooth, the degree of $m_{k}^{\prime \perp}$ on a closed curve in $B_{1}^{\prime}$ is zero, and therefore 


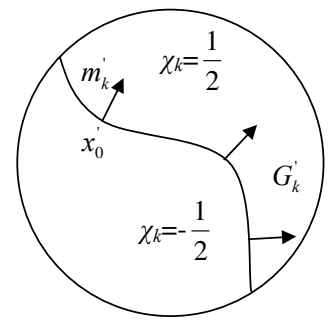

Fig. 10. The orbit $X_{k}$ of the vector field $m_{k}^{\prime} \perp$ passing through $x_{0}^{\prime}$ in the ball $B_{1}^{\prime}$.

an orbit of 65 cannot be closed. Now let $X_{k}$ be the orbit of 65 passing through $x_{0}^{\prime}$ (see Figure 10, i.e.,

$$
\left\{\begin{array}{l}
\dot{X}_{k}(t)=m_{k}^{\prime}\left(X_{k}(t)\right), \\
X_{k}(0)=x_{0}^{\prime}
\end{array}\right.
$$

Then either the orbit $X_{k}$ reaches the boundary $\partial B_{1}^{\prime}$ in finite time, or the limit points of $X_{k}$ (see [4, Chapter 16]) belong to the boundary $\partial B_{1}^{\prime}$ : Suppose that neither of these alternatives holds, i.e., there is a limit point inside the ball $B_{1}^{\prime}$. Since 65 has no critical point, Poincaré-Bendixson's theorem (see [4, Theorem 2.1]) implies that the limit set of $X_{k}$ should contain a periodic orbit, which contradicts the nonexistence of cycles for (65). Hence, the orbit $X_{k}$ separates the ball $B_{1}^{\prime}$ into a right side $G_{k}^{\prime}$ (where $m_{k}^{\prime}$ is the inner normal vector to $\partial G_{k}^{\prime}$ ) and a left side $B_{1}^{\prime} \backslash G_{k}^{\prime}$. We define

$$
\chi_{k}= \begin{cases}1 / 2 & \text { in } G_{k}^{\prime}, \\ -1 / 2 & \text { in } B_{1}^{\prime} \backslash G_{k}^{\prime}\end{cases}
$$

Then $\chi_{k} \in B V_{\text {loc }}\left(B_{1}^{\prime}\right)$ with

$$
D^{\prime} \chi_{k}=m_{k}^{\prime} \mathcal{H}^{1}\left\llcorner X_{k}=m_{k}^{\prime}\left|D^{\prime} \chi_{k}\right|\right.
$$

Moreover, in the ball $B^{\prime}\left(x_{0}^{\prime}, 1-\left|x_{0}^{\prime}\right|\right) \subset B_{1}^{\prime}$ we have, for every $r \in\left(0,1-\left|x_{0}^{\prime}\right|\right)$,

$$
\int_{B^{\prime}\left(x_{0}^{\prime}, r\right)}\left|D^{\prime} \chi_{k}\right|=\mathcal{H}^{1}\left(\left\{X_{k} \in B^{\prime}\left(x_{0}^{\prime}, r\right)\right\}\right) \geq 2 r .
$$

Step 3. The sequence $\left\{\chi_{k}\right\}_{k \in \mathbb{N}}$ is uniformly locally bounded in $B V\left(B_{1}^{\prime}\right)$ and any accumulation point $\chi$ of $\left\{\chi_{k}\right\}_{k \uparrow \infty}$ in $L_{\text {loc }}^{1}\left(B_{1}^{\prime}\right)$ belongs to $B V_{\text {loc }}\left(B_{1}^{\prime},\{-1 / 2,1 / 2\}\right)$. (The jump set of $\chi$ is concentrated on the characteristic of $m^{\prime}$ passing through $x_{0}^{\prime}$, for almost all $x_{0}^{\prime} \in B_{1}^{\prime}$.) It is enough to prove that $\left\{\chi_{k}\right\}_{k \in \mathbb{N}}$ is bounded in $B V\left(B^{\prime}\left(z_{0}^{\prime}, r\right)\right)$ for any $z_{0}^{\prime} \in B_{1}^{\prime}$ such that $B^{\prime}\left(z_{0}^{\prime}, 2 r\right) \subset B_{1}^{\prime}$. We apply Corollary 3 in the ball $B\left(z_{0}, 2 r\right)$ for the restriction of $\left.\chi_{k}\right|_{B^{\prime}\left(z_{0}^{\prime}, r\right)}$ where $z_{0}=\left(z_{0}^{\prime}, 0\right) \in B_{1}$ : 


$$
\begin{aligned}
\left|\int_{B^{\prime}\left(z_{0}^{\prime}, r\right)} \chi_{k} \nabla^{\prime} \cdot m_{k}^{\prime} d x^{\prime}\right| \stackrel{\text { 66] }}{\leq} & \left(\frac{2}{\pi}\left|\ln \varepsilon_{k}\right| \int_{\mathbb{R}^{2}}\left|D^{\prime}\left(\left.\chi_{k}\right|_{B^{\prime}\left(z_{0}^{\prime}, r\right)}\right)\right| \int_{B_{1}}\left|h_{k}\right|^{2} d x\right)^{1 / 2} \\
& +C(r)\left(\frac{1}{\left|\ln \varepsilon_{k}\right|} e_{k}\left(B_{1}\right)\right)^{1 / 2}\left(1+\int_{\mathbb{R}^{2}}\left|D^{\prime}\left(\left.\chi_{k}\right|_{B^{\prime}\left(z_{0}^{\prime}, r\right)}\right)\right|\right) \\
\leq & \frac{1}{2} \int_{\mathbb{R}^{2}}\left|D^{\prime}\left(\left.\chi_{k}\right|_{B^{\prime}\left(z_{0}^{\prime}, r\right)}\right)\right|+\frac{1}{2} e_{k}\left(B_{1}\right) \\
& +C(r)\left(\frac{1}{\left|\ln \varepsilon_{k}\right|} e_{k}\left(B_{1}\right)\right)^{1 / 2}\left(1+\int_{\mathbb{R}^{2}}\left|D^{\prime}\left(\left.\chi_{k}\right|_{B^{\prime}\left(z_{0}^{\prime}, r\right)}\right)\right|\right) \\
\leq & \left(\frac{1}{2}+C(r)\left(\frac{1}{\left|\ln \varepsilon_{k}\right|} e_{k}\left(B_{1}\right)\right)^{1 / 2}\right) \int_{\mathbb{R}^{2}}\left|D^{\prime}\left(\left.\chi_{k}\right|_{B^{\prime}\left(z_{0}^{\prime}, r\right)}\right)\right| \\
& +\frac{1}{2} e_{k}\left(B_{1}\right)+C(r)\left(\frac{1}{\left|\ln \varepsilon_{k}\right|} e_{k}\left(B_{1}\right)\right)^{1 / 2},
\end{aligned}
$$

where $C(r)>0$ denotes a generic constant only depending on $r$ and we used the CauchySchwarz inequality. Since

$$
\int_{\mathbb{R}^{2}}\left|D^{\prime}\left(\left.\chi_{k}\right|_{B^{\prime}\left(z_{0}^{\prime}, r\right)}\right) \stackrel{\text { 66] }}{\leq} \int_{B^{\prime}\left(z_{0}^{\prime}, r\right)}\right| D^{\prime} \chi_{k} \mid+\pi r
$$

we deduce that

$$
\begin{aligned}
\left|\int_{B^{\prime}\left(z_{0}^{\prime}, r\right)} \chi_{k} \nabla^{\prime} \cdot m_{k}^{\prime} d x^{\prime}\right| \leq & \left(\frac{1}{2}+C(r)\left(\frac{1}{\left|\ln \varepsilon_{k}\right|} e_{k}\left(B_{1}\right)\right)^{1 / 2}\right) \int_{B^{\prime}\left(z_{0}^{\prime}, r\right)}\left|D^{\prime} \chi_{k}\right| \\
& +\frac{1}{2} e_{k}\left(B_{1}\right)+C(r)\left(1+\left(\frac{1}{\left|\ln \varepsilon_{k}\right|} e_{k}\left(B_{1}\right)\right)^{1 / 2}\right) .
\end{aligned}
$$

By 67), integration by parts leads to

$$
\int_{B^{\prime}\left(z_{0}^{\prime}, r\right)} \chi_{k} \nabla^{\prime} \cdot m_{k}^{\prime} d x^{\prime}=\int_{\partial B^{\prime}\left(z_{0}^{\prime}, r\right)} \chi_{k}^{-} m_{k}^{\prime} \cdot v d \mathcal{H}^{1}-\int_{B^{\prime}\left(z_{0}^{\prime}, r\right)}\left|D^{\prime} \chi_{k}\right|
$$

where $\chi_{k}^{-}$denotes the interior trace of $\left.\chi_{k}\right|_{\partial B^{\prime}\left(z_{0}^{\prime}, r\right)}$ and $v$ is the unit outer normal vector on $\partial B^{\prime}\left(z_{0}^{\prime}, r\right)$. In combination with $[69$, this yields

$$
\begin{aligned}
\left(\frac{1}{2}-\right. & \left.C(r)\left(\frac{1}{\left|\ln \varepsilon_{k}\right|} e_{k}\left(B_{1}\right)\right)^{1 / 2}\right) \int_{B^{\prime}\left(z_{0}^{\prime}, r\right)}\left|D^{\prime} \chi_{k}\right| \\
& \leq \int_{\partial B^{\prime}\left(z_{0}^{\prime}, r\right)} \chi_{k}^{-} m_{k}^{\prime} \cdot v d \mathcal{H}^{1}+\frac{1}{2} e_{k}\left(B_{1}\right)+C(r)\left(1+\left(\frac{1}{\left|\ln \varepsilon_{k}\right|} e_{k}\left(B_{1}\right)\right)^{1 / 2}\right) \\
& \leq \frac{1}{2} e_{k}\left(B_{1}\right)+C(r)\left(1+\left(\frac{1}{\left|\ln \varepsilon_{k}\right|} e_{k}\left(B_{1}\right)\right)^{1 / 2}\right) .
\end{aligned}
$$


By assumption, as $\varepsilon_{k} \rightarrow 0$ and the sequence $\left\{e_{k}\left(B_{1}\right)\right\}$ is bounded, this estimate implies the boundedness of $\left\{\int_{B^{\prime}\left(z_{0}^{\prime}, r\right)}\left|D^{\prime} \chi_{k}\right|\right\}$. Since $z_{0}^{\prime}$ was arbitrarily chosen, we conclude that $\left\{\chi_{k}\right\}$ is uniformly locally bounded in $B V\left(B_{1}^{\prime}\right)$. Thus, there exists a function $\chi \in B V_{\text {loc }}\left(B_{1}^{\prime},\{-1 / 2,1 / 2\}\right)$ such that up to a subsequence,

$$
\chi_{k} \rightarrow \chi \quad \text { in } L^{1}\left(B_{1}^{\prime}\right)
$$

Step 4. We prove that

$$
-\int_{\mathbb{R}^{2}} \chi m^{\prime} \cdot \nabla^{\prime} \eta^{2} d x^{\prime}+\frac{1}{4 \delta} \int_{\mathbb{R}^{3}} \eta^{2} d e \geq 2(1-\delta) \int_{0}^{\infty} \eta^{2} d r,
$$

for any small $\delta>0$ and any $\eta \in C_{c}^{\infty}\left(B\left(x_{0}, R\right)\right)$ such that $\eta^{2}$ is a decreasing function of $r=\left|x-x_{0}\right|$ only, where $R \in\left(0,1-\left|x_{0}^{\prime}\right|\right]$. For such an $\left.\eta, 68\right)$ implies that

$$
\begin{aligned}
\int_{\mathbb{R}^{2}} \eta^{2}\left|D^{\prime} \chi_{k}\right| & =\int_{0}^{\infty}\left(-\frac{d}{d r} \eta^{2}\right) \int_{B^{\prime}\left(x_{0}^{\prime}, r\right)}\left|D^{\prime} \chi_{k}\right| d r \geq 2 \int_{0}^{\infty}\left(-\frac{d}{d r} \eta^{2}\right) r d r \\
& =2 \int_{0}^{\infty} \eta^{2} d r .
\end{aligned}
$$

Using 67, integration by parts leads to

$$
-\int_{\mathbb{R}^{2}} \chi_{k} m_{k}^{\prime} \cdot \nabla^{\prime} \eta^{2} d x^{\prime}=\int_{\mathbb{R}^{2}} \eta^{2} \chi_{k} \nabla^{\prime} \cdot m_{k}^{\prime} d x^{\prime}+\int_{\mathbb{R}^{2}} \eta^{2}\left|D^{\prime} \chi_{k}\right| .
$$

We apply Corollary 2

$$
\begin{aligned}
\left|\int_{\mathbb{R}^{2}} \eta^{2} \chi_{k} \nabla^{\prime} \cdot m_{k}^{\prime} d x^{\prime}\right| \stackrel{66}{\leq} & \left(\frac{2}{\pi}\left|\ln \varepsilon_{k}\right| \int_{\mathbb{R}^{2}} \eta^{2}\left|D^{\prime} \chi_{k}\right| \int_{B_{1}} \eta^{2}\left|h_{k}\right|^{2} d x\right)^{1 / 2} \\
& +C(R, \eta)\left(\frac{1}{\left|\ln \varepsilon_{k}\right|} e_{k}\left(B_{1}\right)\right)^{1 / 2}\left(1+\int_{\mathbb{R}^{2}}|\eta|\left|D^{\prime} \chi_{k}\right|\right) \\
\leq & \delta \int_{\mathbb{R}^{2}} \eta^{2}\left|D^{\prime} \chi_{k}\right|+\frac{1}{4 \delta} \int_{\mathbb{R}^{3}} \eta^{2} d e_{k} \\
& +C(R, \eta)\left(\frac{1}{\left|\ln \varepsilon_{k}\right|} e_{k}\left(B_{1}\right)\right)^{1 / 2}\left(1+\int_{\mathbb{R}^{2}}|\eta|\left|D^{\prime} \chi_{k}\right|\right),
\end{aligned}
$$

where $C(R, \eta)>0$ denotes a generic constant only depending on $R$ and $\eta$ and we used Young's inequality for some small $\delta>0$. Combined with (72) and (73), this yields

$$
\begin{aligned}
& -\int_{\mathbb{R}^{2}} \chi_{k} m_{k}^{\prime} \cdot \nabla^{\prime} \eta^{2} d x^{\prime}+\frac{1}{4 \delta} \int_{\mathbb{R}^{3}} \eta^{2} d e_{k} \\
& \quad \geq(1-\delta) \int_{\mathbb{R}^{2}} \eta^{2}\left|D^{\prime} \chi_{k}\right|-C(R, \eta)\left(\frac{1}{\left|\ln \varepsilon_{k}\right|} e_{k}\left(B_{1}\right)\right)^{1 / 2}\left(1+\int_{\mathbb{R}^{2}}|\eta|\left|D^{\prime} \chi_{k}\right|\right) \\
& \quad \geq 2(1-\delta) \int_{0}^{\infty} \eta^{2} d r-C(R, \eta)\left(\frac{1}{\left|\ln \varepsilon_{k}\right|} e_{k}\left(B_{1}\right)\right)^{1 / 2}\left(1+\int_{\mathbb{R}^{2}}|\eta|\left|D^{\prime} \chi_{k}\right|\right) .
\end{aligned}
$$


We may pass to the limit $k \rightarrow \infty$ in order to deduce (71). Indeed, by (61) and (70), it follows that

Because of (64), we have

$$
\int_{\mathbb{R}^{2}} \chi_{k} m_{k}^{\prime} \cdot \nabla^{\prime} \eta^{2} d x^{\prime} \rightarrow \int_{\mathbb{R}^{2}} \chi m^{\prime} \cdot \nabla^{\prime} \eta^{2} d x^{\prime}
$$

$$
\frac{1}{4 \delta} \int_{\mathbb{R}^{3}} \eta^{2} d e_{k} \rightarrow \frac{1}{4 \delta} \int_{\mathbb{R}^{3}} \eta^{2} d e .
$$

By assumption (17), Step 3 and $\varepsilon_{k} \rightarrow 0$, the last term in (74) vanishes as $k \rightarrow \infty$.

Step 5. We show that $\left|m^{\prime}\right|=1$ a.e. in $B_{1}^{\prime}$. Let $x_{0}^{\prime} \in B_{1}^{\prime}$ be a Lebesgue point of $m^{\prime}$ and of vanishing $\mathcal{H}^{1}$-density of $e$, i.e.,

$$
\lim _{r \rightarrow 0} \frac{1}{R^{2}} \int_{B^{\prime}\left(x_{0}^{\prime}, R\right)}\left|m^{\prime}\left(x^{\prime}\right)-m^{\prime}\left(x_{0}^{\prime}\right)\right| d x^{\prime}=0 \quad \text { and } \quad \limsup _{R \rightarrow 0} \frac{e\left(B\left(x_{0}, R\right)\right)}{R}=0
$$

where $x_{0}=\left(x_{0}^{\prime}, 0\right)$. (By the Lebesgue decomposition theorem and Vitali covering lemma, almost every point in $B_{1}^{\prime}$ has the above properties.) By (62) and (75), it follows that $\left|m^{\prime}\left(x_{0}^{\prime}\right)\right| \leq 1$. In order to prove that $\left|m^{\prime}\left(x_{0}^{\prime}\right)\right|=1$, we show that $\left|m^{\prime}\left(x_{0}^{\prime}\right)\right| \geq 1$. For that, we now specify $\eta^{2}$ in 71$]$ :

$$
\eta^{2}(r)=\eta_{R}^{2}(r):=\frac{1}{R} \eta_{1}^{2}\left(\frac{r}{R}\right), \quad \forall r \in(0, R),
$$

where $\eta_{1}^{2} \in C_{c}^{\infty}\left(B\left(x_{0}, 1\right)\right)$ is a decreasing function of $r=\left|x-x_{0}\right|$ only. Since $\eta_{R}^{2} \leq C / R$ and $\left|\nabla^{\prime} \eta_{R}^{2}\right| \leq C / R^{2}$, by (75) we have

$$
\lim _{R \rightarrow 0} \int_{\mathbb{R}^{3}} \eta_{R}^{2} d e=0, \quad \lim _{R \rightarrow 0}\left|\int_{\mathbb{R}^{2}} \chi m^{\prime} \cdot \nabla^{\prime} \eta_{R}^{2} d x^{\prime}-\int_{\mathbb{R}^{2}} \chi m^{\prime}\left(x_{0}^{\prime}\right) \cdot \nabla^{\prime} \eta_{R}^{2} d x^{\prime}\right|=0 .
$$

Hence, from (71) we obtain

$$
-\limsup _{R \rightarrow 0} \int_{\mathbb{R}^{2}} \chi m^{\prime}\left(x_{0}^{\prime}\right) \cdot \nabla^{\prime} \eta_{R}^{2} d x^{\prime} \geq 2(1-\delta) \lim _{R \rightarrow 0} \int_{0}^{\infty} \eta_{R}^{2} d r=2(1-\delta) \int_{0}^{\infty} \eta_{1}^{2} d r .
$$

Since $\delta>0$ was arbitrary, this leads to

$$
-\limsup _{R \rightarrow 0} \int_{\mathbb{R}^{2}} \chi m^{\prime}\left(x_{0}^{\prime}\right) \cdot \nabla^{\prime} \eta_{R}^{2} d x^{\prime} \geq 2 \int_{0}^{\infty} \eta_{1}^{2} d r .
$$

On the other hand, we have

$$
\begin{aligned}
\left|\int_{\mathbb{R}^{2}} \chi m^{\prime}\left(x_{0}^{\prime}\right) \cdot \nabla^{\prime} \eta_{R}^{2} d x^{\prime}\right| & \leq\left|m^{\prime}\left(x_{0}^{\prime}\right)\right| \int_{\mathbb{R}^{2}}\left|\frac{\partial}{\partial x_{1}} \eta_{R}^{2}\right| d x^{\prime}=\left|m^{\prime}\left(x_{0}^{\prime}\right)\right| \int_{\mathbb{R}^{2}}\left|\frac{\partial}{\partial x_{1}} \eta_{1}^{2}\right| d x^{\prime} \\
& =\left|m^{\prime}\left(x_{0}^{\prime}\right)\right| \int_{0}^{\infty}\left(-\frac{d}{d r} \eta_{1}^{2}\right) r d r \int_{0}^{2 \pi}|\cos \theta| d \theta \\
& =2\left|m^{\prime}\left(x_{0}^{\prime}\right)\right| \int_{0}^{\infty} \eta_{1}^{2} d r .
\end{aligned}
$$


Thus, from 76 we obtain

$$
2\left|m^{\prime}\left(x_{0}^{\prime}\right)\right| \int_{0}^{\infty} \eta_{1}^{2} d r \geq 2 \int_{0}^{\infty} \eta_{1}^{2} d r,
$$

i.e., $\left|m^{\prime}\left(x_{0}^{\prime}\right)\right| \geq 1$.

Step 6. End of proof. Let now $m^{\prime}$ be an accumulation point of the sequence $\left\{m_{k}^{\prime}\right\}_{k \uparrow \infty}$ in $L_{\text {loc }}^{1}\left(B_{1}^{\prime}\right)$. Since $\left|m_{k}^{\prime}\right|=1$, we deduce that $\left|m^{\prime}\right|=1$ a.e. in $B_{1}^{\prime}$. By (17), $\int_{B_{1}}\left|h_{k}\right|^{2} d x \rightarrow 0$ as $k \rightarrow \infty$, and therefore (16) yields

$$
\lim _{k \rightarrow \infty} \int_{B_{1}^{\prime}} \zeta \nabla^{\prime} \cdot m_{k}^{\prime} d x^{\prime}=0, \quad \forall \zeta \in C_{c}^{\infty}\left(B_{1}^{\prime}\right) .
$$

Thus, $\nabla^{\prime} \cdot m^{\prime}=0$ distributionally in $B_{1}^{\prime}$.

\section{Zero-energy states}

Recall that a zero-energy state is an accumulation point $m^{\prime}: B_{1}^{\prime} \rightarrow S^{1}$ of a sequence of magnetizations $\left\{m_{k}^{\prime}: B_{1}^{\prime} \rightarrow S^{1}\right\}_{k \uparrow \infty}$ in $L_{\text {loc }}^{1}\left(B_{1}^{\prime}\right)$ such that 19\} holds for a sequence $\varepsilon_{k} \rightarrow 0$ and some stray fields $\left\{h_{k}: B_{1} \rightarrow \mathbb{R}^{3}\right\}_{k \uparrow \infty}$ related to $\left\{m_{k}^{\prime}\right\}$ by (16). In order to prove Theorem 5, we proceed in several steps. A key ingredient to Theorem 5 is the following additional property of limits $m^{\prime}$ :

Lemma 1. Together with (18), any accumulation point $m^{\prime}: B_{1}^{\prime} \rightarrow \mathbb{R}^{2}$ of $\left\{m_{k}^{\prime}\right\}_{k \uparrow \infty}$ in $L^{1}\left(B_{1}^{\prime}\right)$ has the following property: For all $x_{0}^{\prime} \in B_{1}^{\prime}$ there exists $\chi: B_{1}^{\prime} \rightarrow\{-1 / 2,1 / 2\}$ such that

$$
\begin{gathered}
\nabla^{\prime} \cdot\left(\chi m^{\prime}\right)=\left|D^{\prime} \chi\right| \quad \text { distributionally in } B_{1}^{\prime}, \\
\int_{B^{\prime}\left(x_{0}^{\prime}, r\right)}\left|D^{\prime} \chi\right| \geq 2 r \quad \text { for all } 0<r<1-\left|x_{0}^{\prime}\right| .
\end{gathered}
$$

Proof. Fix $x_{0}^{\prime} \in B_{1}^{\prime}$. Let $\left\{\chi_{k}\right\}$ be defined in $B_{1}^{\prime}$ as in Step 2 of the proof of Theorem 4 (see 66). By Step 3 of that proof,

$$
\left\{\int_{B_{r}^{\prime}}\left|D^{\prime} \chi_{k}\right|\right\}_{k \uparrow \infty} \text { is bounded for all } 0<r<1 .
$$

Hence, after passage to a subsequence, we may assume that there exists $\chi: B_{1}^{\prime} \rightarrow$ $\{-1 / 2,1 / 2\}$ of locally bounded variation such that

$$
\chi_{k} \rightarrow \chi \quad \text { in } L^{1}\left(B_{1}^{\prime}\right) .
$$

It remains to argue that $\chi$ satisfies (77) and (78). For a given $\zeta \in C_{c}^{\infty}\left(B_{1}^{\prime}\right)$, we shall establish the following four statements:

$$
-\int_{B_{1}^{\prime}} \chi_{k} \nabla^{\prime} \zeta \cdot m_{k}^{\prime} d x^{\prime}-\int_{B_{1}^{\prime}} \zeta\left|D^{\prime} \chi_{k}\right| \rightarrow 0,
$$




$$
\begin{array}{ll}
-\int_{B_{1}^{\prime}} \chi \nabla^{\prime} \zeta \cdot m^{\prime} d x^{\prime}-\int_{B_{1}^{\prime}} \zeta\left|D^{\prime} \chi\right| \geq 0 & \text { if } \zeta \geq 0, \\
-\int_{B_{1}^{\prime}} \chi \nabla^{\prime} \zeta \cdot m^{\prime} d x^{\prime}-\int_{B_{1}^{\prime}} \zeta\left|D^{\prime} \chi\right| \leq 0 & \text { if } \zeta \geq 0 \\
\int_{B_{1}^{\prime}} \zeta\left|D^{\prime} \chi_{k}\right| \rightarrow \int_{B_{1}^{\prime}} \zeta\left|D^{\prime} \chi\right| & \text { if } \zeta \geq 0 .
\end{array}
$$

In order to establish (81), we will use again the identity 67) based on the construction of $\chi_{k}$, i.e., $m_{k}^{\prime} \cdot D^{\prime} \chi_{k}=\left|D^{\prime} \chi_{k}\right|$; namely,

$$
\begin{aligned}
& -\int_{B_{1}^{\prime}} \nabla^{\prime} \zeta \cdot m_{k}^{\prime} \chi_{k} d x^{\prime}-\int_{B_{1}^{\prime}} \zeta\left|D^{\prime} \chi_{k}\right| \\
& =\int_{B_{1}^{\prime}} \zeta \chi_{k} \nabla^{\prime} \cdot m_{k}^{\prime} d x^{\prime}+\int_{B_{1}^{\prime}} \zeta m_{k}^{\prime} \cdot D^{\prime} \chi_{k}-\int_{B_{1}^{\prime}} \zeta\left|D^{\prime} \chi_{k}\right|=\int_{B_{1}^{\prime}} \zeta \chi_{k} \nabla^{\prime} \cdot m_{k}^{\prime} d x^{\prime} .
\end{aligned}
$$

The second ingredient is Corollary 1 , applied for the function $\zeta \chi_{k}$ in the ball $B_{1}^{\prime}$ and $d=\operatorname{dist}\left(\operatorname{supp} \zeta, \partial B_{1}^{\prime}\right)$. Since $\sup \left|\chi_{k}\right|=1 / 2$, we obtain

$$
\begin{aligned}
\left|\int_{\mathbb{R}^{2}}\left(\zeta \chi_{k}\right) \nabla^{\prime} \cdot m_{k}^{\prime} d x^{\prime}\right| \leq & \left(\frac{2}{\pi}\left|\ln \varepsilon_{k}\right| \sup |\zeta| \int_{B_{1}^{\prime}}\left|D^{\prime}\left(\zeta \chi_{k}\right)\right| \int_{B_{1}}\left|h_{k}\right|^{2} d x\right)^{1 / 2} \\
& +\frac{C}{d}\left(\varepsilon_{k} \int_{B_{1}^{\prime}}\left|\nabla^{\prime} \cdot m_{k}^{\prime}\right|^{2} d x^{\prime}+\int_{B_{1}}\left|h_{k}\right|^{2} d x\right)^{1 / 2} \\
& \times\left(\sup |\zeta|+\int_{B_{1}^{\prime}}\left|D^{\prime}\left(\zeta \chi_{k}\right)\right|\right) .
\end{aligned}
$$

As $\left|D^{\prime}\left(\zeta \chi_{k}\right)\right| \leq \frac{1}{2}\left|\nabla^{\prime} \zeta\right|+|\zeta|\left|D^{\prime} \chi_{k}\right|$, by $\left[79\right.$ we deduce that the sequence $\left\{\int_{B_{1}^{\prime}}\left|D^{\prime}\left(\zeta \chi_{k}\right)\right|\right\}$ is bounded, and from 190 it follows that

$$
\int_{B_{1}^{\prime}} \zeta \chi_{k} \nabla^{\prime} \cdot m_{k}^{\prime} d x^{\prime} \rightarrow 0 \quad \text { as } k \rightarrow \infty
$$

Now (81) follows from (85) and 86). Statement (82) follows easily from 81). Indeed, from (80) and $m_{k}^{\prime} \rightarrow m^{\prime}$ in $L^{1}\left(B_{1}^{\prime}\right)$, we have

$$
\int_{B_{1}^{\prime}} \chi_{k} \nabla^{\prime} \zeta \cdot m_{k}^{\prime} d x^{\prime} \rightarrow \int_{B_{1}^{\prime}} \chi \nabla^{\prime} \zeta \cdot m^{\prime} d x^{\prime}
$$

on the other hand, the lower semicontinuity of $\left|D^{\prime} \chi_{k}\right|$ under 80 implies

$$
\int_{B_{1}^{\prime}} \zeta\left|D^{\prime} \chi\right| \leq \liminf _{k \rightarrow \infty} \int_{B_{1}^{\prime}} \zeta\left|D^{\prime} \chi_{k}\right| \quad \text { if } \zeta \geq 0 \text { in } B_{1}^{\prime}
$$


Statement 83 is a general fact which follows from 18$\}$. Indeed, let $\left\{m_{\delta}^{\prime}\right\}_{\delta \downarrow}$ denote the mollification of $m^{\prime}$ by convolution. For any $r<1$ and sufficiently small $\delta$, we then have, in a classical sense,

$$
\nabla^{\prime} \cdot m_{\delta}^{\prime}=0 \quad \text { and } \quad\left|m_{\delta}^{\prime}\right|^{2} \leq 1 \quad \text { in } B_{r}^{\prime}
$$

Therefore,

$$
\begin{aligned}
\int_{B_{1}^{\prime}} \chi \nabla^{\prime} \zeta \cdot m_{\delta}^{\prime} d x^{\prime} & \stackrel{88}{=} \int_{B_{1}^{\prime}} \chi \nabla^{\prime} \cdot\left(\zeta m_{\delta}^{\prime}\right) d x^{\prime} \\
& =-\int_{B_{1}^{\prime}} \zeta m_{\delta}^{\prime} \cdot D^{\prime} \chi \stackrel{88}{\leq} \int_{B_{1}^{\prime}} \zeta\left|D^{\prime} \chi\right| \quad \text { if } \zeta \geq 0
\end{aligned}
$$

Statement 84 is a straightforward consequence of the previous ones:

$$
\begin{aligned}
\lim _{k \rightarrow \infty} \int_{B_{1}^{\prime}} \zeta\left|D^{\prime} \chi_{k}\right| & \stackrel{81}{=}-\lim _{k \rightarrow \infty} \int_{B_{1}^{\prime}} \chi_{k} \nabla^{\prime} \zeta \cdot m_{k}^{\prime} d x^{\prime} \\
& =\int_{B_{1}^{\prime}} \chi \nabla^{\prime} \zeta \cdot m^{\prime} d x^{\prime 82,83} \int_{B_{1}^{\prime}} \zeta\left|D^{\prime} \chi\right|,
\end{aligned}
$$

if $\zeta \geq 0$.

We now argue that (77) and (78) are true. We start with (77). From 82 ) and $(83)$, we already know that

$$
-\int_{B_{1}^{\prime}} \chi \nabla^{\prime} \zeta \cdot m^{\prime} d x^{\prime}=\int_{B_{1}^{\prime}} \zeta\left|D^{\prime} \chi\right| \quad \text { for all } \zeta \in C_{c}^{\infty}\left(B_{1}^{\prime}\right) \text { with } \zeta \geq 0 .
$$

Since any $\zeta \in C_{c}^{\infty}\left(B_{1}^{\prime}\right)$ can be approximated both in $H^{1}\left(B_{1}^{\prime}\right)$ and $C_{c}\left(B_{1}^{\prime}\right)$ by $\zeta_{\delta}$ 's of the form

$$
\zeta_{\delta}=\zeta_{\delta}^{+}-\zeta_{\delta}^{-} \quad \text { with positive and negative parts } \zeta_{\delta}^{+}, \zeta_{\delta}^{-} \in C_{c}^{\infty}\left(B_{1}^{\prime}\right)
$$

(89) implies (77). An approximation of the form 90 can be constructed as $\zeta_{\delta}=\phi_{\delta}(\zeta)$, where $\left\{\phi_{\delta}\right\}_{\delta \downarrow 0} \subset C^{\infty}(\mathbb{R})$ is an approximation of the identity with the following properties:

$$
\phi_{\delta}(t)=0 \text { for }|t| \leq \delta, \quad \frac{d \phi_{\delta}}{d t}(t) \rightarrow 1 \text { for } t \neq 0, \quad\left|\frac{d \phi_{\delta}}{d t}(t)\right| \leq 1 \text { for all } t .
$$

We now address (78). Fix $0<r<1-\left|x_{0}^{\prime}\right|$. We will derive (78) from the corresponding property 68 of $\chi_{k}$ and (84). Let $\left\{\eta_{\delta}\right\}_{\delta \downarrow 0} \subset C_{c}^{\infty}\left(B_{1}^{\prime}\right)$ be an approximation of the characteristic function $1_{B^{\prime}\left(x_{0}^{\prime}, r\right)}$ in the following sense:

$$
\begin{gathered}
\eta_{\delta}\left(x^{\prime}\right)=0 \text { for } x^{\prime} \notin B^{\prime}\left(x_{0}^{\prime}, r\right), \quad \eta_{\delta}\left(x^{\prime}\right)=1 \text { for } x^{\prime} \in B^{\prime}\left(x_{0}^{\prime}, r-\delta\right), \\
0 \leq \eta_{\delta}\left(x^{\prime}\right) \leq 1 \text { for } x^{\prime} \in B_{1}^{\prime} .
\end{gathered}
$$


We have

$$
\begin{aligned}
\int_{B^{\prime}\left(x_{0}^{\prime}, r\right)}\left|D^{\prime} \chi\right| & \geq \int_{B_{1}^{\prime}} \eta_{\delta}\left|D^{\prime} \chi\right| \stackrel{84]}{=} \lim _{k \rightarrow \infty} \int_{B_{1}^{\prime}} \eta_{\delta}\left|D^{\prime} \chi_{k}\right| \geq \liminf _{k \rightarrow \infty} \int_{B^{\prime}\left(x_{0}^{\prime}, r-\delta\right)}\left|D^{\prime} \chi_{k}\right| \\
& \stackrel{68}{\geq} 2(r-\delta),
\end{aligned}
$$

and we deduce 78 by letting $\delta \rightarrow 0$.

The next lemma establishes that the $\chi$ 's from Lemma 1 are minimal (perimeter minimizing). It is a well-known general fact that sets whose normal can be extended to a divergence-free unit-length vector field are minimal.

Lemma 2. Let $\chi: B_{1}^{\prime} \rightarrow\{-1 / 2,1 / 2\}$ have the property (77) for some $m^{\prime}: B_{1}^{\prime} \rightarrow S^{1}$ with

$$
\nabla^{\prime} \cdot m^{\prime}=0 \quad \text { distributionally in } B_{1}^{\prime} .
$$

Then $\chi$ is minimal in $B_{1}^{\prime}$ in the sense that for any function $\tilde{\chi}: B_{1}^{\prime} \rightarrow\{-1 / 2,1 / 2\}$ with $\operatorname{supp}(\tilde{\chi}-\chi) \subset \subset B_{1}^{\prime}$, we have

$$
\left|D^{\prime} \chi\right|\left(B_{1}^{\prime}\right) \leq\left|D^{\prime} \tilde{\chi}\right|\left(B_{1}^{\prime}\right) .
$$

Proof. Let $0<r<1$ be such that $\operatorname{supp}(\tilde{\chi}-\chi) \subset B_{r}^{\prime}$. Select a $\zeta \in C_{c}^{\infty}\left(B_{1}^{\prime}\right)$ with $\zeta=1$ in $B_{r}^{\prime}$ and $\zeta \geq 0$ in $B_{1}^{\prime}$. Then we have

$$
\begin{aligned}
\left|D^{\prime} \chi\right|\left(B_{r}^{\prime}\right)-\left|D^{\prime} \tilde{\chi}\right|\left(B_{r}^{\prime}\right) & =\int_{B_{1}^{\prime}} \zeta\left|D^{\prime} \chi\right|-\int_{B_{1}^{\prime}} \zeta\left|D^{\prime} \tilde{\chi}\right| \\
& \stackrel{777}{=}-\int_{B_{1}^{\prime}} \chi \nabla^{\prime} \zeta \cdot m^{\prime} d x^{\prime}-\int_{B_{1}^{\prime}} \zeta\left|D^{\prime} \tilde{\chi}\right| \\
& =-\int_{B_{1}^{\prime}} \tilde{\chi} \nabla^{\prime} \zeta \cdot m^{\prime} d x^{\prime}-\int_{B_{1}^{\prime}} \zeta\left|D^{\prime} \tilde{\chi}\right| .
\end{aligned}
$$

The argument used to establish the inequality 83 in the proof of Lemma 1 also yields this lemma (with $\chi$ replaced by $\tilde{\chi}$ ).

For convenience of the reader, the following lemma gives an elementary proof for the fact that minimal sets in two dimensions are locally half-spaces.

Lemma 3. Let $\chi: B_{1}^{\prime} \rightarrow\{-1 / 2,1 / 2\}$ satisfy

$$
\begin{gathered}
\chi \text { is minimal in } B_{1}^{\prime}, \\
\int_{B_{r}^{\prime}}\left|D^{\prime} \chi\right| \geq 2 r \quad \text { for all } r \in(0,1) .
\end{gathered}
$$

Then $\chi$ is the characteristic function of a centered half-space in $B_{1-\pi / 4}^{\prime}$ (see Figure 11, i.e., there exists $v \in S^{1}$ such that

$$
\chi=\left\{\begin{array}{ll}
1 / 2 & \text { for } x^{\prime} \cdot v>0 \\
-1 / 2 & \text { else }
\end{array}\right\} \quad \mathcal{L}^{2} \text {-a.e. in } B_{1-\pi / 4}^{\prime} .
$$






Fig. 11. The characteristic $\chi$ in the ball $B_{1-\pi / 4}^{\prime}$.

Proof. We start by arguing that

$$
\left|D^{\prime} \chi\right|\left(B_{1}^{\prime}\right) \leq \pi
$$

Let $0<r<1$ be arbitrary. We compare $\chi$ to $\tilde{\chi}_{+}, \tilde{\chi}_{-}$given by

$$
\tilde{\chi}_{+}=\left\{\begin{array}{ll}
1 / 2 & \text { in } B_{r}^{\prime}, \\
\chi & \text { else, }
\end{array} \quad \tilde{\chi}_{-}= \begin{cases}-1 / 2 & \text { in } B_{r}^{\prime}, \\
\chi & \text { else. }\end{cases}\right.
$$

By assumption 91, we obtain

$$
\begin{aligned}
\left|D^{\prime} \chi\right|\left(B_{r}^{\prime}\right) \leq \min \left\{\left|D^{\prime} \tilde{\chi}_{-}\right|\left(B_{r}^{\prime}\right)+\int_{\partial B_{r}^{\prime}}\left|\chi^{-}-\tilde{\chi}_{-}^{-}\right| d \mathcal{H}^{1}\right. \\
\\
\left.\left|D^{\prime} \tilde{\chi}_{+}\right|\left(B_{r}^{\prime}\right)+\int_{\partial B_{r}^{\prime}}\left|\chi^{-}-\tilde{\chi}_{+}^{-}\right| d \mathcal{H}^{1}\right\},
\end{aligned}
$$

where $\chi^{-}, \tilde{\chi}_{-}^{-}$and $\tilde{\chi}_{+}^{-}$denote the interior traces of $\left.\chi\right|_{\partial B_{r}^{\prime}},\left.\tilde{\chi}_{-}\right|_{\partial B_{r}^{\prime}}$ and $\left.\tilde{\chi}_{+}\right|_{\partial B_{r}^{\prime}}$ respectively. In view of the form of $\tilde{\chi}_{-}, \tilde{\chi}_{+}$, this turns into

$$
\begin{aligned}
\left|D^{\prime} \chi\right|\left(B_{r}^{\prime}\right) & \leq \min \left\{\int_{\partial B_{r}^{\prime}}\left|\chi^{-}+1 / 2\right| d \mathcal{H}^{1}, \int_{\partial B_{r}^{\prime}}\left|\chi^{-}-1 / 2\right| d \mathcal{H}^{1}\right\} \\
& =\min \left\{\pi r+\int_{\partial B_{r}^{\prime}} \chi^{-} d \mathcal{H}^{1}, \pi r-\int_{\partial B_{r}^{\prime}} \chi^{-} d \mathcal{H}^{1}\right\} \leq \pi r .
\end{aligned}
$$

From this, we deduce (93) by monotone convergence as $r \uparrow 1$.

We now argue that there exists an $r \in[1-\pi / 4,1)$ such that

$$
\int_{\partial B_{r}^{\prime}}\left|D_{\theta} \chi^{-}\right| \in\{0,2\}
$$

where $\int_{\partial B_{r}^{\prime}}\left|D_{\theta} \chi^{-}\right|$denotes the total variation of the trace $\chi^{-}$on $\partial B_{r}^{\prime}$. Indeed,

$$
\begin{aligned}
\mathcal{L}^{1}\left(\left\{r \in(0,1): \int_{\partial B_{r}^{\prime}}\left|D_{\theta} \chi^{-}\right| \geq 4\right\}\right) & \leq \frac{1}{4} \int_{0}^{1}\left(\int_{\partial B_{r}^{\prime}}\left|D_{\theta} \chi^{-}\right|\right) d r \\
& \leq \frac{1}{4}\left|D^{\prime} \chi\right|\left(B_{1}^{\prime}\right) \stackrel{93}{\leq} \frac{\pi}{4}
\end{aligned}
$$


Hence, there exists $1-\pi / 4 \leq r<1$ such that

$$
\int_{\partial B_{r}^{\prime}}\left|D_{\theta} \chi^{-}\right|<4
$$

But since $\chi^{-} \in\{-1 / 2,1 / 2\}$, we have $\int_{\partial B_{r}^{\prime}}\left|D_{\theta} \chi^{-}\right| \in\{0,2,4, \ldots\}$, so that 95 entails 94 .

We now argue that there exists $v \in S^{1}$ such that

$$
\chi^{-}=\left\{\begin{array}{ll}
1 / 2 & \text { for } x^{\prime} \cdot v>0 \\
-1 / 2 & \text { else }
\end{array}\right\} \quad \mathcal{H}^{1} \text {-a.e. on } \partial B_{r}^{\prime},
$$

where $r$ is as in (94). Indeed, by (94), there exist $v \in S^{1}$ and $\alpha \in \mathbb{R}$ such that

$$
\chi^{-}=\left\{\begin{array}{ll}
1 / 2 & \text { for } x^{\prime} \cdot v>\alpha \\
-1 / 2 & \text { else }
\end{array}\right\} \quad \mathcal{H}^{1} \text {-a.e. on } \partial B_{r}^{\prime}
$$

(see Figure 12). We compare $\chi$ with $\tilde{\chi}$ given by

$$
\tilde{\chi}= \begin{cases}1 / 2 & \text { for } x^{\prime} \cdot v>\alpha \text { and } x^{\prime} \in B_{r}^{\prime} \\ -1 / 2 & \text { for } x^{\prime} \cdot v \leq \alpha \text { and } x^{\prime} \in B_{r}^{\prime} \\ \chi & \text { else }\end{cases}
$$

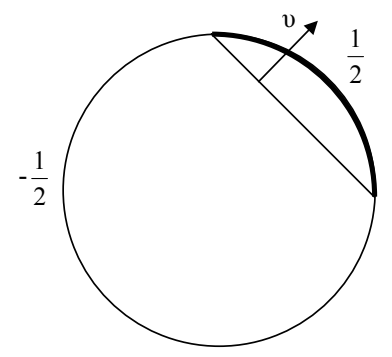

Fig. 12. The trace $\chi^{-}$on $\partial B_{r}^{\prime}$.

By (97), the traces of $\left.\chi\right|_{\partial B_{r}^{\prime}}$ and $\left.\tilde{\chi}\right|_{\partial B_{r}^{\prime}}$ coincide. Hence, by the assumption (91),

$$
\left|D^{\prime} \chi\right|\left(B_{r}^{\prime}\right) \leq\left|D^{\prime} \tilde{\chi}\right|\left(B_{r}^{\prime}\right) \text {. }
$$

Because of assumption 92 this yields

$$
2 r \leq \mathcal{H}^{1}\left(\left\{x^{\prime} \cdot v=\alpha\right\} \cap B_{r}^{\prime}\right),
$$

which enforces $\alpha=0$ so that 97 turns into 96. We finally argue that

$$
\chi=\left\{\begin{array}{ll}
1 / 2 & \text { for } x^{\prime} \cdot v>0 \\
-1 / 2 & \text { else }
\end{array}\right\} \quad \mathcal{L}^{2} \text {-a.e. in } B_{r}^{\prime},
$$


where $v$ is as in 96. Indeed, 96 implies that

$$
\int_{B_{r}^{\prime}} v \cdot D^{\prime} \chi=\int_{\partial B_{r}^{\prime}} v \cdot \frac{x^{\prime}}{r} \chi^{-} d \mathcal{H}^{1}=2 r
$$

whereas 98 yields

$$
\left|D^{\prime} \chi\right|\left(B_{r}^{\prime}\right) \leq \mathcal{H}^{1}\left(\left\{x^{\prime} \cdot v=0\right\} \cap B_{r}^{\prime}\right) \leq 2 r .
$$

Hence we necessarily have

$$
D^{\prime} \chi=v\left|D^{\prime} \chi\right| \quad\left|D^{\prime} \chi\right| \text {-a.e. in } B_{r}^{\prime} .
$$

Since $\chi \in\{-1 / 2,1 / 2\}$, this implies that

$$
\chi=\left\{\begin{array}{ll}
1 / 2 & \text { for } x^{\prime} \cdot v>\alpha \\
-1 / 2 & \text { else }
\end{array}\right\} \quad \mathcal{L}^{2} \text {-a.e. in } B_{r}^{\prime},
$$

for some $\alpha \in \mathbb{R}$. Since the trace $\chi^{-}$is given by [96, $\chi$ must indeed be of the form 99 ].

The next lemma establishes that the characteristic functions from Lemma 1 are locally ordered.

Lemma 4. Let $m^{\prime}: B_{1}^{\prime} \rightarrow \mathbb{R}^{2}$ satisfy 18 . Let $\chi_{0}: B_{1}^{\prime} \rightarrow\{-1 / 2,1 / 2\}$ have the properties:

- $\chi_{0}$ is the characteristic function of a centered half-space, i.e., there exists $v_{0} \in S^{1}$ such that

$$
\chi_{0}=\left\{\begin{array}{ll}
1 / 2 & \text { for } x^{\prime} \cdot v_{0}>0 \\
-1 / 2 & \text { else }
\end{array}\right\} \quad \text { in } B_{1}^{\prime} ;
$$

- $\chi_{0}$ satisfies (77).

Let $\chi: B_{1}^{\prime} \rightarrow\{-1 / 2,1 / 2\}$ have the properties:

- $\chi$ is the characteristic function of a half-space, i.e., there exist $\nu \in S^{1}$ and $\alpha \in \mathbb{R}$ such that

$$
\chi=\left\{\begin{array}{ll}
1 / 2 & \text { for } x^{\prime} \cdot v>\alpha \\
-1 / 2 & \text { else }
\end{array}\right\} \quad \text { in } B_{1}^{\prime}
$$

- $\chi$ satisfies $(77)$.

Then $\chi \leq \chi_{0}$ in $B_{1-\pi / 4}^{\prime}$ or $\chi \geq \chi_{0}$ in $B_{1-\pi / 4}^{\prime}$.

Proof. We distinguish three cases.

Case 1: $\mathcal{H}^{0}\left(\left\{x^{\prime} \cdot v_{0}=0\right\} \cap\left\{x^{\prime} \cdot v=\alpha\right\}\right) \leq 1$ and $\alpha \leq 0$. In this case, we consider $\tilde{\chi}$ given by

$$
\tilde{\chi}=\left\{\begin{array}{ll}
1 / 2 & \text { for } x^{\prime} \cdot v_{0}>0 \text { and } x^{\prime} \cdot v>\alpha \\
-1 / 2 & \text { else }
\end{array}\right\} \quad \text { in } B_{1}^{\prime}
$$



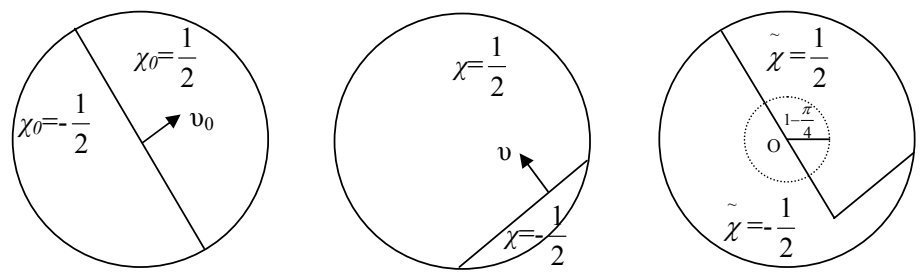

Fig. 13. The characteristics $\chi_{0}, \chi$ and $\tilde{\chi}$ in the ball $B_{1}^{\prime}$.

(see Figure 13). We argue that

$$
\begin{array}{cl}
\nabla^{\prime} \cdot\left(\tilde{\chi} m^{\prime}\right)=\left|D^{\prime} \tilde{\chi}\right| & \text { distributionally in } B_{1}^{\prime}, \\
\int_{B_{r}^{\prime}}\left|D^{\prime} \tilde{\chi}\right| \geq 2 r & \text { for all } r \in(0,1) .
\end{array}
$$

Indeed, 100 ) holds distributionally in

- $B_{1}^{\prime} \cap\left\{x^{\prime} \cdot v_{0}>0\right\}$, since there $\tilde{\chi}=\chi$, so $(100)$ follows from the property (77) of $\chi$;

- $B_{1}^{\prime} \cap\left\{x^{\prime} \cdot v_{0}<0\right\}$, since there $\tilde{\chi}=-1 / 2$, so (100) follows from [18);

- $B_{1}^{\prime} \cap\left\{x^{\prime} \cdot v>\alpha\right\}$, since there $\tilde{\chi}=\chi_{0}$, so (100) follows from the property (77) of $\chi_{0}$;

- $B_{1}^{\prime} \cap\left\{x^{\prime} \cdot v<\alpha\right\}$, since there $\tilde{\chi}=-1 / 2$, so (100) follows from [18].

Hence, (100) holds distributionally in $B_{1}^{\prime} \backslash\left(\left\{x^{\prime} \cdot v_{0}=0\right\} \cap\left\{x^{\prime} \cdot v=\alpha\right\}\right)$. By assumption, $\left\{x^{\prime} \cdot v_{0}=0\right\} \cap\left\{x^{\prime} \cdot v=\alpha\right\}$ consists of at most a single point. But (100) is insensitive to sets of vanishing $\mathcal{H}^{1}$-measure. This establishes (100). The inequality (101) follows from the fact that $0 \in \partial\left(\left\{x^{\prime} \cdot v_{0}>0\right\} \cap\left\{x^{\prime} \cdot v>\alpha\right\}\right)$, which is a consequence of our assumption $\alpha \leq 0$. According to Lemma 2, (18) and (100) imply that $\tilde{\chi}$ is minimal in $B_{1}^{\prime}$. According to Lemma 3 , this and (101) imply that $\tilde{\chi}$ is the characteristic function of a centered halfspace in $B_{1-\pi / 4}^{\prime}$. Hence $\left\{x^{\prime} \cdot v_{0}>0\right\} \cap\left\{x^{\prime} \cdot v>\alpha\right\}$ is a centered half-space in $B_{1-\pi / 4}^{\prime}$. In view of $\alpha \leq 0$, this yields

$$
\left\{x^{\prime} \cdot v_{0}>0\right\} \cap\left\{x^{\prime} \cdot v>\alpha\right\} \cap B_{1-\pi / 4}^{\prime}=\left\{x^{\prime} \cdot v_{0}>0\right\} \cap B_{1-\pi / 4}^{\prime},
$$

that is,

$$
x^{\prime} \cdot v>\alpha \quad \text { in }\left\{x^{\prime} \cdot v_{0}>0\right\} \cap B_{1-\pi / 4}^{\prime},
$$

whence $\chi \geq \chi_{0}$ in $B_{1-\pi / 4}^{\prime}$.

Case 2: $\mathcal{H}^{0}\left(\left\{x^{\prime} \cdot v_{0}=0\right\} \cap\left\{x^{\prime} \cdot v=\alpha\right\}\right) \leq 1$ and $\alpha \geq 0$. In this case, we consider $\tilde{\chi}$ given by

$$
\tilde{\chi}=\left\{\begin{array}{ll}
1 / 2 & \text { for } x^{\prime} \cdot v_{0}>0 \text { or } x^{\prime} \cdot v>\alpha \\
-1 / 2 & \text { else }
\end{array}\right\} \quad \text { in } B_{1}^{\prime}
$$

and we argue as before to arrive at $\chi \leq \chi_{0}$ in $B_{1-\pi / 4}^{\prime}$. 
Case 3: $\mathcal{H}^{0}\left(\left\{x^{\prime} \cdot v_{0}=0\right\} \cap\left\{x^{\prime} \cdot v=\alpha\right\}\right)>1$. In this case, we necessarily have

$$
\alpha=0 \quad \text { and } \quad\left(\nu=v_{0} \text { or } v=-v_{0}\right) .
$$

In the case of $v=v_{0}$, we have $\chi=\chi_{0}$. The case of $v=-v_{0}$ cannot occur since then

$$
\chi_{0}+\chi=0 \quad \mathcal{L}^{2} \text {-a.e. in } B_{1}^{\prime},
$$

so that (77) would yield

$$
\left|D^{\prime} \chi_{0}\right|+\left|D^{\prime} \chi\right|=\nabla^{\prime} \cdot\left(\chi_{0} m^{\prime}\right)+\nabla^{\prime} \cdot\left(\chi m^{\prime}\right)=0
$$

in particular $D^{\prime} \chi_{0}=0$, which is a contradiction.

The next lemma establishes Lipschitz continuity of $m^{\prime}$ locally in $B_{1}^{\prime}$. Because of translation and scaling invariance, it suffices to prove the following:

Lemma 5. Let $m^{\prime}$ be as in Lemma 1 Let 0 and $y^{\prime} \in B_{1}^{\prime}$ be Lebesgue points of $m^{\prime}$. Then

$$
\left|m^{\prime}\left(y^{\prime}\right)-m^{\prime}(0)\right| \leq \frac{2 \sqrt{2}}{(1-\pi / 4)^{2}}\left|y^{\prime}\right| \quad \text { for all } y^{\prime} \in B_{\frac{1}{2}(1-\pi / 4)^{2}}^{\prime} .
$$

Proof. Let $\chi_{0}$ and $\chi$ denote the characteristic functions associated to 0 and $y^{\prime}$ respectively, according to Lemma 1. According to Lemmas 2 and 3 there exist $v_{0}, v \in S^{1}$ such that

$$
\begin{gathered}
\chi_{0}=\left\{\begin{array}{ll}
1 / 2 & \text { for } x^{\prime} \cdot v_{0}>0 \\
-1 / 2 & \text { else }
\end{array}\right\} \quad \text { in } B_{1-\pi / 4}^{\prime}, \\
\chi=\left\{\begin{array}{ll}
1 / 2 & \text { for }\left(x^{\prime}-y^{\prime}\right) \cdot v>0 \\
-1 / 2 & \text { else }
\end{array}\right\} \quad \text { in } B^{\prime}\left(y^{\prime},(1-\pi / 4)\left(1-\left|y^{\prime}\right|\right)\right) .
\end{gathered}
$$

Since

$$
\left|y^{\prime}\right| \leq \frac{1}{2}(1-\pi / 4)^{2} \leq \frac{\frac{1}{2}(1-\pi / 4)}{2-\pi / 4}
$$

we have

$$
B^{\prime}\left(y^{\prime},(1-\pi / 4)\left(1-\left|y^{\prime}\right|\right)\right) \supset B^{\prime}\left(0,(1-\pi / 4)\left(1-\left|y^{\prime}\right|\right)-\left|y^{\prime}\right|\right) \supset B_{\frac{1}{2}(1-\pi / 4)}^{\prime},
$$

so that both 102 and 103 hold in $B_{\frac{1}{2}(1-\pi / 4)}^{\prime}$. Thus an application of Lemma 4 yields

$$
\chi \leq \chi_{0} \text { in } B_{\frac{1}{2}(1-\pi / 4)^{2}}^{\prime} \quad \text { or } \quad \chi \geq \chi_{0} \text { in } B_{\frac{1}{2}(1-\pi / 4)^{2}}^{\prime} .
$$

We can consider only the first alternative, that is,

$$
\left\{x^{\prime} \cdot v_{0} \leq 0\right\} \cap B_{\frac{1}{2}(1-\pi / 4)^{2}}^{\prime} \subset\left\{\left(x^{\prime}-y^{\prime}\right) \cdot v \leq 0\right\} .
$$

Thus, $v \cdot v_{0}>0$. We introduce the abbreviations

$$
\delta:=\frac{y^{\prime} \cdot v}{r}, \quad r:=\frac{1}{2}(1-\pi / 4)^{2} .
$$




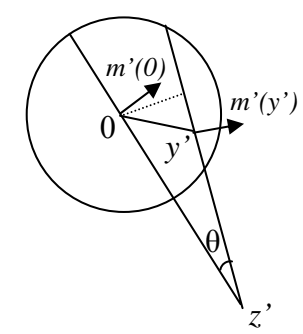

Fig. 14. Geometry of characteristics.

By elementary geometry (see Figure 14, this implies

$$
\left|v-v_{0}\right|^{2} \leq 2 \delta^{2} .
$$

Indeed, if $v=v_{0}$, then 104 is obvious. Otherwise, $v \neq v_{0}$, and therefore the point of intersection $z^{\prime}$ of the two lines respectively orthogonal to $v_{0}$ and $v$ and passing through 0 and $y^{\prime}$, lies outside the ball $B_{r}^{\prime}$; denoting by $\theta=\angle\left(\nu, \nu_{0}\right) \in(0, \pi / 2]$ the angle between $v$ and $v_{0}$, it follows that

$$
\frac{y^{\prime} \cdot v}{\sin \theta}=\left|z^{\prime}\right| \geq r \quad \text { and } \quad \cos \frac{\theta}{2} \geq \frac{\sqrt{2}}{2},
$$

that is,

$$
\delta \geq \sin \theta=2 \sin \frac{\theta}{2} \cos \frac{\theta}{2} \geq \sqrt{2} \sin \frac{\theta}{2}=\left|v-v_{0}\right| \frac{1}{\sqrt{2}} .
$$

Hence,

$$
\left|v-v_{0}\right| \leq \frac{2 \sqrt{2}}{(1-\pi / 4)^{2}}\left|y^{\prime}\right| .
$$

It remains to prove that (77) implies

$$
v=m^{\prime}\left(y^{\prime}\right) \text { and } \quad v_{0}=m^{\prime}(0) .
$$

We just show the latter. Indeed, in view of (102), (77) takes the form

$$
\frac{1}{2} \int_{\left\{x^{\prime} \cdot \nu_{0}<0\right\}} m^{\prime} \cdot \nabla^{\prime} \zeta d x^{\prime}-\frac{1}{2} \int_{\left\{x^{\prime} \cdot v_{0}>0\right\}} m^{\prime} \cdot \nabla^{\prime} \zeta d x^{\prime}=\int_{\left\{x^{\prime} \cdot \nu_{0}=0\right\}} \zeta d \mathcal{H}^{1}
$$

for all $\zeta \in C_{c}^{\infty}\left(B_{1-\pi / 4}^{\prime}\right)$. We now fix a $\zeta_{1} \in C_{c}^{\infty}\left(B_{1-\pi / 4}^{\prime}\right)$ such that $\int_{\left\{x^{\prime} \cdot v_{0}=0\right\}} \zeta_{1} d \mathcal{H}^{1}=1$ and for $r<1$, consider $\zeta_{r} \in C_{c}^{\infty}\left(B_{r(1-\pi / 4)}^{\prime}\right)$ given by

$$
\zeta_{r}\left(x^{\prime}\right)=\frac{1}{r} \zeta_{1}\left(\frac{x^{\prime}}{r}\right)
$$

Since

$$
\int_{\mathbb{R}^{2}}\left|\nabla^{\prime} \zeta_{r}\right| d x^{\prime}=\int_{\mathbb{R}^{2}}\left|\nabla^{\prime} \zeta_{1}\right| d x^{\prime}
$$


and 0 is a Lebesgue point of $m^{\prime}$, we have

$$
\begin{aligned}
\lim _{r \rightarrow 0}\left(\frac{1}{2} \int_{\left\{x^{\prime} \cdot v_{0}<0\right\}} m^{\prime} \cdot \nabla^{\prime} \zeta_{r} d x^{\prime}-\frac{1}{2} \int_{\left\{x^{\prime} \cdot v_{0}>0\right\}} m^{\prime} \cdot \nabla^{\prime} \zeta_{r} d x^{\prime}\right) \\
=m^{\prime}(0) \cdot \lim _{r \rightarrow 0}\left(\frac{1}{2} \int_{\left\{x^{\prime} \cdot v_{0}<0\right\}} \nabla^{\prime} \zeta_{r} d x^{\prime}-\frac{1}{2} \int_{\left\{x^{\prime} \cdot v_{0}>0\right\}} \nabla^{\prime} \zeta_{r} d x^{\prime}\right) \\
=\left(m^{\prime}(0) \cdot v_{0}\right) \lim _{r \rightarrow 0} \int_{\left\{x^{\prime} \cdot v_{0}=0\right\}} \zeta_{r} d \mathcal{H}^{1} .
\end{aligned}
$$

Since

$$
\int_{\left\{x^{\prime} \cdot v_{0}=0\right\}} \zeta_{r} d \mathcal{H}^{1}=\int_{\left\{x^{\prime} \cdot v_{0}=0\right\}} \zeta_{1} d \mathcal{H}^{1}=1,
$$

from (106) and (107) we obtain $m^{\prime}(0) \cdot v_{0}=1$, which implies (105) because $\left|m^{\prime}(0)\right|=1$.

The last lemma establishes the principle of characteristics for $m^{\prime}$ in $B_{1}^{\prime}$. By translation and scaling invariance and a continuity argument, it suffices to prove the following:

Lemma 6. Let $m^{\prime}$ be as in Lemma 1 and Lipschitz continuous. Then

$$
m^{\prime}\left(m^{\prime}(0)^{\perp}\right)=m^{\prime}(0) \quad \text { for all }|t|<1-\pi / 4 .
$$

Proof. Let $\chi$ be the characteristic function associated to 0 according to Lemma 1 . From Lemmas 2 and 3 we gather that there exists $v \in S^{1}$ such that

$$
\chi=\left\{\begin{array}{ll}
1 / 2 & \text { for } x^{\prime} \cdot v>0 \\
-1 / 2 & \text { else }
\end{array}\right\} \quad \text { in } B_{1-\pi / 4}^{\prime} .
$$

As in Lemma 5 we deduce from (77) and the continuity of $m^{\prime}$ that

$$
m^{\prime}=v \quad \text { on }\left\{x^{\prime} \cdot v=0\right\} \cap B_{1-\pi / 4}^{\prime} .
$$

This is a reformulation of 108 .

\section{Optimality of the straight walls}

In this section, we prove Theorem 1 .

Proof of Theorem 1. Let $m^{\prime}: \mathbb{R}^{2} \rightarrow S^{1}$ and $h: \mathbb{R}^{3} \rightarrow \mathbb{R}^{3}$ satisfy the hypothesis of Theorem 11. Using the same argument as in the proof of Theorem 4, and (1), we identify the center line of the transition layer: There exists a set $G^{\prime} \subset \mathbb{R}^{2}$ with inner normal $v^{\prime}$ such that

$$
\begin{aligned}
& G^{\prime} \text { is } L \text {-periodic in } x_{2}, \\
& (1,+\infty) \times \mathbb{R} \subset G^{\prime}, \quad(-\infty,-1) \times \mathbb{R} \subset \mathbb{R}^{2} \backslash G^{\prime}, \\
& m^{\prime}=v^{\prime} \quad \text { on } \partial G^{\prime}
\end{aligned}
$$




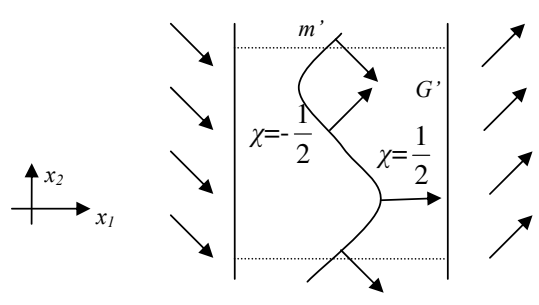

Fig. 15. Center line of the wall.

(see Figure 15]. This set was already introduced in [5]. We consider the related characteristic function

$$
\chi= \begin{cases}1 / 2 & \text { in } G^{\prime} \\ -1 / 2 & \text { in } \mathbb{R}^{2} \backslash G^{\prime}\end{cases}
$$

Then (109) translates into

$$
\begin{aligned}
& \chi \text { is } L \text {-periodic in } x_{2} \\
& \chi= \pm 1 / 2 \text { for } \pm x_{1} \geq 1 \\
& \int_{\mathbb{R} \times[0, L)} \eta^{2} \chi \nabla^{\prime} \cdot m^{\prime} d x^{\prime}=-\int_{\mathbb{R} \times[0, L)} \nabla^{\prime}\left(\eta^{2}\right) \cdot m^{\prime} \chi d x^{\prime}-\int_{\mathbb{R} \times[0, L)} \eta^{2}\left|D^{\prime} \chi\right|
\end{aligned}
$$

where $\eta \in C^{\infty}\left(\mathbb{R}^{3}\right)$ is an $L$-periodic function in $x_{2}$ that satisfies 55 . As in 63), we introduce the energy density $e$ as a non-negative measure on $\mathbb{R}^{3}$ via

$$
\int_{\mathbb{R}^{3}} \zeta d e=\frac{2}{\pi}|\ln \varepsilon|\left(\varepsilon \int_{\mathbb{R}^{2}} \zeta\left|\nabla^{\prime} \cdot m^{\prime}\right|^{2} d x^{\prime}+\int_{\mathbb{R}^{3}} \zeta|h|^{2} d x\right), \quad \forall \zeta \in C_{c}\left(\mathbb{R}^{3}\right)
$$

Step 1. We have an a priori bound on $L^{-1} \int_{\mathbb{R} \times[0, L)}\left|D^{\prime} \chi\right|$ in terms of $L^{-1} e(\mathbb{R} \times[0, L)$ $\times \mathbb{R})$ : for any $\alpha \in(0,1)$,

$$
\begin{aligned}
& (1-\alpha) L^{-1} \int_{\mathbb{R} \times[0, L)}\left|D^{\prime} \chi\right| \leq m_{1, \infty}+\frac{1}{4 \alpha} L^{-1} e(\mathbb{R} \times[0, L) \times \mathbb{R}) \\
& \quad+C(L)\left(|\ln \varepsilon|^{-1} e(\mathbb{R} \times[0, L) \times \mathbb{R})\right)^{1 / 2}\left(1+\int_{\mathbb{R} \times[0, L)}\left|D^{\prime} \chi\right|\right) .
\end{aligned}
$$

Indeed, with the above choices and notations, 577 turns into

$$
\begin{aligned}
& \left|\int_{\mathbb{R} \times[0, L)} \eta^{2} \chi \nabla^{\prime} \cdot m^{\prime} d x^{\prime}\right| \stackrel{\sqrt[112]{\leq}}{\leq}\left(\int_{\mathbb{R} \times[0, L) \times \mathbb{R}} \eta^{2} d e \int_{\mathbb{R} \times[0, L)} \eta^{2}\left|D^{\prime} \chi\right|\right)^{1 / 2} \\
& +C(L)\left(|\ln \varepsilon|^{-1} e(\mathbb{R} \times[0, L) \times \mathbb{R})\right)^{1 / 2}\left(\sup _{\mathbb{R}^{3}}|\eta|+\sup _{\mathbb{R}^{3}}|\nabla \eta|\right) \cdot\left(\sup _{\mathbb{R}^{3}}|\eta|+\int_{\mathbb{R} \times[0, L)}|\eta|\left|D^{\prime} \chi\right|\right) .
\end{aligned}
$$


Using (113) for the left-hand side and Young's inequality for the first term of the righthand side yields, for any $\alpha \in(0,1)$,

$$
\begin{aligned}
(1-\alpha) \int_{\mathbb{R} \times[0, L)} \eta^{2}\left|D^{\prime} \chi\right| \leq & -\int_{\mathbb{R} \times[0, L)} \nabla^{\prime}\left(\eta^{2}\right) \cdot m^{\prime} \chi d x^{\prime}+\frac{1}{4 \alpha} \int_{\mathbb{R} \times[0, L) \times \mathbb{R}} \eta^{2} d e \\
& +C(L)\left(|\ln \varepsilon|^{-1} e(\mathbb{R} \times[0, L) \times \mathbb{R})\right)^{1 / 2} \\
\times & \left(\sup _{\mathbb{R}^{3}}|\eta|+\sup _{\mathbb{R}^{3}}|\nabla \eta|\right) \cdot\left(\sup _{\mathbb{R}^{3}}|\eta|+\int_{\mathbb{R} \times[0, L)}|\eta|\left|D^{\prime} \chi\right|\right) .
\end{aligned}
$$

We select $\eta: \mathbb{R}^{3} \rightarrow \mathbb{R}$ such that

$$
\begin{aligned}
& \eta=\eta\left(x_{1}, x_{3}\right), \eta=1 \quad \text { on }(-1,1) \times \mathbb{R} \times\{0\}, \\
& \operatorname{supp} \eta \subset(-2,2) \times \mathbb{R} \times(-1,1), \quad|\eta| \leq 1, \quad|\nabla \eta| \leq C .
\end{aligned}
$$

We consider the terms in 116 one-by-one:

$$
\begin{gathered}
\int_{\mathbb{R} \times[0, L)} \eta^{2}\left|D^{\prime} \chi\right| \stackrel{112[117]}{=} \int_{\mathbb{R} \times[0, L)}\left|D^{\prime} \chi\right| \\
-\int_{\mathbb{R} \times[0, L)} \nabla^{\prime}\left(\eta^{2}\right) \cdot m^{\prime} \chi d x^{\prime} \stackrel{1112] 117]}{-}-\int_{(-\infty,-1) \times[0, L)}\left(\begin{array}{c}
\partial_{1} \eta^{2} \\
0
\end{array}\right) \cdot\left(\begin{array}{c}
m_{1, \infty} \\
-\sqrt{1-m_{1, \infty}^{2}}
\end{array}\right) \frac{1}{2} d x^{\prime} \\
\quad-\int_{(1,+\infty) \times[0, L)}\left(\begin{array}{c}
\partial_{1} \eta^{2} \\
0
\end{array}\right) \cdot\left(\begin{array}{c}
m_{1, \infty} \\
\sqrt{1-m_{1, \infty}^{2}}
\end{array}\right) \frac{1}{2} d x^{\prime} \\
\quad=L m_{1, \infty} \\
\int_{\mathbb{R} \times[0, L) \times \mathbb{R}} \eta^{2} d e \leq e(\mathbb{R} \times[0, L) \times \mathbb{R}) .
\end{gathered}
$$

Using (117) to estimate the $\eta$-terms in (116), we then obtain

$$
\begin{aligned}
(1-\alpha) \int_{\mathbb{R} \times[0, L)}\left|D^{\prime} \chi\right| \leq & L m_{1, \infty}+\frac{1}{4 \alpha} e(\mathbb{R} \times[0, L) \times \mathbb{R}) \\
& +C(L)\left(|\ln \varepsilon|^{-1} e(\mathbb{R} \times[0, L) \times \mathbb{R})\right)^{1 / 2}\left(1+\int_{\mathbb{R} \times[0, L)}\left|D^{\prime} \chi\right|\right) .
\end{aligned}
$$

Dividing by $L$ yields 115 .

Step 2. Sketch of the proof of Theorem 1, We argue by contradiction. Consider sequences $\left\{\varepsilon_{k}\right\}_{k \in \mathbb{N}} \subset(0, \infty)$ with $\varepsilon_{k} \downarrow 0,\left\{m_{k}^{\prime}: \mathbb{R}^{2} \rightarrow S^{1}\right\}_{k \uparrow \infty}$ and $\left\{h_{k}: \mathbb{R}^{3} \rightarrow \mathbb{R}^{3}\right\}_{k \uparrow \infty}$ that satisfy the first three hypotheses in Theorem 1 and

$$
\limsup _{k \rightarrow \infty} L^{-1} e_{k}(\mathbb{R} \times[0, L) \times \mathbb{R}) \leq\left(1-m_{1, \infty}\right)^{2},
$$


which corresponds to (12) (here, $e_{k}$ is the energy density (114) associated to $m_{k}^{\prime}$ and $h_{k}$ ). By periodicity of $e_{k}$, 119 implies that the energy is locally bounded, so that we may apply Theorem 4 Hence there exists a measurable $m^{\prime}: \mathbb{R}^{2} \rightarrow S^{1}$ with

$$
m_{k}^{\prime} \rightarrow m^{\prime} \quad \text { in } L_{\mathrm{loc}}^{1}\left(\mathbb{R}^{2}\right)
$$

after passage to a subsequence. Properties (1) and (4) are preserved under (120) while in addition (see Theorem 4),

$$
\nabla^{\prime} \cdot m^{\prime}=0 \quad \text { distributionally in } \mathbb{R}^{2} .
$$

By (1) and (4), (120) yields

$$
\int_{\mathbb{R} \times[0, L)}\left|m_{k}^{\prime}-m^{\prime}\right| d x^{\prime} \rightarrow 0 .
$$

We thus have to argue that $m^{\prime}$ has the form (14). By periodicity of $e,(119)$ implies that there exists a non-negative measure $e$ on $\mathbb{R}^{3}$ such that

$$
e_{k} \rightarrow e \quad \text { weak }^{*} \text { in } \mathcal{M}\left(\mathbb{R}^{3}\right)
$$

after passage to a subsequence. Notice that (119) is preserved under (122):

$$
L^{-1} e(\mathbb{R} \times[0, L) \times \mathbb{R}) \leq\left(1-m_{1, \infty}\right)^{2} .
$$

We shall prove that there exists an $x_{1}^{*} \in[-1,1]$ such that

$$
\operatorname{supp} e \cap((-2,2) \times \mathbb{R} \times(-1,1)) \subseteq\left\{x_{1}^{*}\right\} \times \mathbb{R} \times\{0\} .
$$

We then apply Theorem 5 on balls in $\left(-2, x_{1}^{*}\right) \times \mathbb{R} \times(-1,1)$ and $\left(x_{1}^{*}, 2\right) \times \mathbb{R} \times(-1,1)$ respectively. This shows that $m^{\prime}$ is locally Lipschitz and satisfies the principle of characteristics in both $\left(-2, x_{1}^{*}\right) \times \mathbb{R} \times(-1,1)$ and $\left(x_{1}^{*}, 2\right) \times \mathbb{R} \times(-1,1)$. In view of the form (1), this indeed implies that $m^{\prime}$ is of the form (14). Hence it suffices to show (124).

Step 3. Proof of (124). We first address the function $\chi_{k}$ defined as in 110 for $m_{k}^{\prime}$. In view of (115) (applied to $\chi_{k}$ and $e_{k}$ ) and (119),

$$
\left\{L^{-1} \int_{\mathbb{R} \times[0, L)}\left|D^{\prime} \chi_{k}\right|\right\}_{k \uparrow \infty} \text { is bounded. }
$$

By periodicity (111), there exists a measurable function $\chi: \mathbb{R}^{2} \rightarrow\{-1 / 2,1 / 2\}$ of locally bounded variation such that

$$
\chi_{k} \rightarrow \chi \quad \text { in } L_{\text {loc }}^{1}\left(\mathbb{R}^{2}\right)
$$

up to a subsequence. Notice that (111) and the boundary conditions (112) are preserved by 126 . We shall prove in Step 4 that $\chi$ is of the form

$$
\chi= \pm 1 / 2 \text { for } \pm x_{1}> \pm x_{1}^{*}
$$


for some $x_{1}^{*} \in[-1,1]$. Now we show how (127) implies (124]. For this we return to 116. Again, by the convergences (120), 1122, ,126) and the boundedness expressed in (119) and 125, inequality (116) (applied for $\chi_{k}, m_{k}^{\prime}$ and $e_{k}$ ) yields, in the limit as $k \rightarrow \infty$,

$$
(1-\alpha) \int_{\mathbb{R} \times[0, L)} \eta^{2}\left|D^{\prime} \chi\right| \leq-\int_{\mathbb{R} \times[0, L)} \nabla^{\prime}\left(\eta^{2}\right) \cdot m^{\prime} \chi d x^{\prime}+\frac{1}{4 \alpha} \int_{\mathbb{R} \times[0, L) \times \mathbb{R}} \eta^{2} d e
$$

for any $\eta \in C^{\infty}\left(\mathbb{R}^{3}\right)$ that is $L$-periodic in $x_{2}$ and satisfies 55 . We choose

$$
\alpha=\frac{1-m_{1, \infty}}{2}
$$

In view of 128,

$$
\begin{aligned}
& \int_{\mathbb{R} \times[0, L) \times \mathbb{R}} \zeta d \lambda \\
& \quad=\frac{1}{4 \alpha} \int_{\mathbb{R} \times[0, L) \times \mathbb{R}} \zeta d e-\int_{\mathbb{R} \times[0, L)} \nabla^{\prime} \zeta \cdot m^{\prime} \chi d x^{\prime}-(1-\alpha) \int_{\mathbb{R} \times[0, L)} \zeta\left|D^{\prime} \chi\right|
\end{aligned}
$$

defines a non-negative distribution $\lambda$ in $(-2,2) \times \mathbb{R} \times(-1,1)$ for functions $\zeta: \mathbb{R}^{3} \rightarrow \mathbb{R}$ which are $L$-periodic in $x_{2}$ and satisfy (55). By (127), $\lambda$ simplifies to

$$
\begin{aligned}
\int_{\mathbb{R} \times[0, L) \times \mathbb{R}} \zeta d \lambda=\frac{1}{4 \alpha} \int_{\mathbb{R} \times[0, L) \times \mathbb{R}} \zeta d e+\frac{1}{2} \int_{\left(-\infty, x_{1}^{*}\right) \times[0, L)} \nabla^{\prime} \zeta \cdot m^{\prime} d x^{\prime} \\
-\frac{1}{2} \int_{\left(x_{1}^{*},+\infty\right) \times[0, L)} \nabla^{\prime} \zeta \cdot m^{\prime} d x^{\prime}-(1-\alpha) \int_{[0, L)} \zeta\left(x_{1}^{*}, x_{2}, 0\right) d x_{2} .
\end{aligned}
$$

In fact, $\lambda$ is a non-negative measure: By $\left|m^{\prime}\right|=1$ and the divergence-free property (121), we have

$$
\left|\frac{1}{2} \int_{\left(-\infty, x_{1}^{*}\right) \times[0, L)} \nabla^{\prime} \zeta \cdot m^{\prime} d x^{\prime}-\frac{1}{2} \int_{\left(x_{1}^{*},+\infty\right) \times[0, L)} \nabla^{\prime} \zeta \cdot m^{\prime} d x^{\prime}\right| \leq \int_{[0, L)}\left|\zeta\left(x_{1}^{*}, x_{2}, 0\right)\right| d x_{2} .
$$

Estimate (131) formally follows from integration by parts and can be rigorously established by approximating $m^{\prime}$ with smooth $m^{\prime}$ 's while preserving $\left|m^{\prime}\right| \leq 1,(121)$ and the periodicity in $x_{2}$. We now consider $\zeta=\eta^{2}$ in (130) such that 55) holds and

$$
\eta=\eta\left(x_{1}, x_{3}\right), \quad \eta=1 \quad \text { on }(-1,1) \times \mathbb{R} \times\{0\}, \quad|\eta| \leq 1 .
$$

Using the same arguments as in (118, we find that 130 turns into

$$
\int_{\mathbb{R} \times[0, L) \times \mathbb{R}} \eta^{2} d \lambda=\frac{1}{4 \alpha} \int_{\mathbb{R} \times[0, L) \times \mathbb{R}} \eta^{2} d e+L m_{1, \infty}-L(1-\alpha) .
$$


Since (123) implies that $\int_{\mathbb{R} \times[0, L) \times \mathbb{R}} \eta^{2} d e \leq e(\mathbb{R} \times[0, L) \times \mathbb{R}) \leq L\left(1-m_{1, \infty}\right)^{2}$, this yields

$$
\int_{\mathbb{R} \times[0, L) \times \mathbb{R}} \eta^{2} d \lambda \leq L\left[\frac{1}{4 \alpha}\left(1-m_{1, \infty}\right)^{2}+m_{1, \infty}-(1-\alpha)\right] \stackrel{[129]}{=} 0 .
$$

We let $\eta^{2}$ converge monotonically to 1 in $(-2,2) \times \mathbb{R} \times(-1,1)$ to obtain

$$
\lambda((-2,2) \times[0, L) \times(-1,1)) \leq 0,
$$

and thus $\lambda \equiv 0$ in $(-2,2) \times[0, L) \times(-1,1)$. Hence, $(130)$ simplifies to

$$
\begin{aligned}
\frac{1}{4 \alpha} \int_{\mathbb{R} \times[0, L) \times \mathbb{R}} \zeta d e= & (1-\alpha) \int_{[0, L)} \zeta\left(x_{1}^{*}, x_{2}, 0\right) d x_{2} \\
& -\frac{1}{2} \int_{\left(-\infty, x_{1}^{*}\right) \times[0, L)} \nabla^{\prime} \zeta \cdot m^{\prime} d x^{\prime}+\frac{1}{2} \int_{\left(x_{1}^{*},+\infty\right) \times[0, L)} \nabla^{\prime} \zeta \cdot m^{\prime} d x^{\prime} \\
& \stackrel{131]}{\leq}(1-\alpha) \int_{[0, L)} \zeta\left(x_{1}^{*}, x_{2}, 0\right) d x_{2}+\int_{[0, L)}\left|\zeta\left(x_{1}^{*}, x_{2}, 0\right)\right| d x_{2}
\end{aligned}
$$

for every $\zeta \in C^{\infty}\left(\mathbb{R}^{3}\right)$ that is $L$-periodic in $x_{2}$ and satisfies 55 . This implies 124 by periodicity of $e$. Thus, it remains to prove (127).

Step 4. Proof of (127). We first notice that by (119), (125) and the lower semicontinuity of $\int_{\mathbb{R} \times[0, L)}\left|D^{\prime} \chi_{k}\right|$ under (126), inequality 115) (applied for $\chi_{k}$ and $e_{k}$ ) yields, in the limit as $k \rightarrow \infty$,

$$
(1-\alpha) L^{-1} \int_{\mathbb{R} \times[0, L)}\left|D^{\prime} \chi\right| \leq m_{1, \infty}+\frac{\left(1-m_{1, \infty}\right)^{2}}{4 \alpha} .
$$

As before, the choice (129) gives

$$
L^{-1} \int_{\mathbb{R} \times[0, L)}\left|D^{\prime} \chi\right| \leq 1 .
$$

Now the boundary conditions (112) and inequality (132) enforce the form (127). For the convenience of the reader, we display this standard argument. Let $\mu$ and $v^{\prime}$ be the measure-theoretic line measure $\left|D^{\prime} \chi\right|$ and normal $D^{\prime} \chi /\left|D^{\prime} \chi\right|$ related to the function $\chi$ of bounded variation. Both inherit the periodicity of $\chi$ and are characterized by

$$
-\int_{\mathbb{R} \times[0, L)} \nabla^{\prime} \cdot \zeta^{\prime} \chi d x^{\prime}=\int_{\mathbb{R} \times[0, L)} v^{\prime} \cdot \zeta^{\prime} d \mu
$$

for all $\zeta^{\prime}: \mathbb{R}^{2} \rightarrow \mathbb{R}^{2}$ which are $L$-periodic in $x_{2}$ and compactly supported in $x_{1}$. Now we show that 112 yields

$$
\int_{\mathbb{R} \times[0, L)} \nu_{1} d \mu=L
$$


Indeed, 134 can be seen by selecting a function $\eta=\eta\left(x_{1}\right)$ with $\eta=1$ for $\left|x_{1}\right| \leq 1$ and $\operatorname{supp} \eta \subset(-2,2) \times \mathbb{R}$ so that

$$
\begin{aligned}
\int_{\mathbb{R} \times[0, L)} v_{1} d \mu & =\int_{\mathbb{R} \times[0, L)} \eta^{2} v_{1} d \mu \stackrel{\sqrt{133}-}{=}-\int_{\mathbb{R} \times[0, L)} \frac{d \eta^{2}}{d x_{1}} \chi d x^{\prime} \\
& =-\int_{(-\infty,-1) \times[0, L)} \frac{-1}{2} \frac{d \eta^{2}}{d x_{1}} d x^{\prime}-\int_{(1,+\infty) \times[0, L)} \frac{1}{2} \frac{d \eta^{2}}{d x_{1}} d x^{\prime}=L .
\end{aligned}
$$

Now (132) (i.e., $\left.\int_{\mathbb{R} \times[0, L)} d \mu \leq L\right)$ and (134) combine to give $\int_{\mathbb{R} \times[0, L)}\left(1-v_{1}\right) d \mu \leq 0$. But since $1-v_{1} \geq 0$ we must have $1-v_{1}=0 \mu$-a.e., that is, $v=\left(\begin{array}{l}1 \\ 0\end{array}\right) \mu$-a.e. Hence 133 turns into

$$
-\int_{\mathbb{R} \times[0, L)} \nabla^{\prime} \cdot \zeta^{\prime} \chi d x^{\prime}=\int_{\mathbb{R} \times[0, L)} \zeta_{1} d \mu .
$$

Choosing $\zeta^{\prime}$ with $\zeta_{1} \equiv 0$, we deduce that $\chi$ has a representative with $\chi=\chi\left(x_{1}\right)$. In particular, (135) then yields

$$
-\int_{\mathbb{R}} \frac{d \eta^{2}}{d x_{1}} \chi d x_{1} \geq 0
$$

for all $\eta=\eta\left(x_{1}\right)$ with compact support. Hence $\chi$ has a representative with $\chi=\chi\left(x_{1}\right)$ that is non-decreasing. Since $\chi \in\{-1 / 2,1 / 2\}$, this yields (127). Now the proof of the theorem is complete.

Remark 4. One can improve (124) to supp e $\subset\left\{x_{1}^{*}\right\} \times \mathbb{R} \times\{0\}$ using Corollary 4 for test functions $\eta$ with support in $(-a, a) \times \mathbb{R} \times(-a, a)$, where $a$ is arbitrarily large.

\section{The case of 1-d magnetizations}

In the framework of Theorem 11, we will now focus on 1-d magnetizations $m^{\prime}=$ $\left(m_{1}\left(x_{1}\right), m_{2}\left(x_{1}\right)\right)$. As in [5], we consider the minimal stray field energy corresponding to $m^{\prime}$ in the strip $\mathbb{R} \times[0,1)$. (Here, we fix the width $L=1$ of the strip.) Let $U \in H_{0}^{1}(\mathbb{R} \times(0,1) \times \mathbb{R})$ be the unique solution of the variational problem:

$$
\int_{\mathbb{R} \times(0,1) \times \mathbb{R}} \nabla U \cdot \nabla \zeta d x=\int_{\mathbb{R} \times(0,1)} \zeta \nabla^{\prime} \cdot m^{\prime} d x^{\prime}, \quad \forall \zeta \in C_{c}^{\infty}(\mathbb{R} \times(0,1) \times \mathbb{R}) .
$$

(That is a direct application of the Lax-Milgram theorem.) The function $U$ is the map in $H_{0}^{1}(\mathbb{R} \times(0,1) \times \mathbb{R})$ that is symmetric in $x_{3}$ and satisfies

$$
\begin{cases}\Delta U=0 & \text { in } \mathbb{R} \times(0,1) \times(\mathbb{R} \backslash\{0\}), \\ {\left[\partial U / \partial x_{3}\right]=-\nabla^{\prime} \cdot m^{\prime}} & \text { on } \mathbb{R} \times(0,1)\end{cases}
$$

where $[\xi]$ denotes the jump of the quantity $\xi$ across the plane $\mathbb{R}^{2} \times\{0\}$. We extend $U$ : $\mathbb{R}^{3} \rightarrow \mathbb{R}$ by 1 -periodicity in the $x_{2}$-direction. Then (2) holds for $h=\nabla U$. An elementary 
computation shows that the stray field energy is given by the homogeneous $H^{-1 / 2}$ norm of the divergence of $m^{\prime}$ :

$$
\int_{\mathbb{R} \times(0,1) \times \mathbb{R}}|\nabla U|^{2} d x=\left.\left.\frac{1}{2} \int_{\mathbb{R} \times(0,1)}|| \nabla^{\prime}\right|^{-1 / 2} \nabla^{\prime} \cdot m^{\prime}\right|^{2} d x^{\prime} .
$$

Since $m^{\prime}$ is $1-\mathrm{d}$, we have

$$
\left.\left.\int_{\mathbb{R} \times(0,1)}|| \nabla^{\prime}\right|^{-1 / 2} \nabla^{\prime} \cdot m^{\prime}\right|^{2} d x^{\prime}=\left.\left.\int_{\mathbb{R}}|| \frac{d}{d x_{1}}\right|^{1 / 2} m_{1}\right|^{2} d x_{1},
$$

and therefore (137) explains the expression of the energy $E_{\varepsilon}^{1 d}\left(m^{\prime}\right)$ given in (11). Also observe that the chosen stray field energy is minimal because for any $h: \mathbb{R}^{3} \rightarrow \mathbb{R}^{3}$ that is 1-periodic in $x_{2}$ and satisfies (2) for $\nabla^{\prime} \cdot m^{\prime}$, we have

$$
\int_{\mathbb{R} \times(0,1) \times \mathbb{R}}|\nabla U|^{2} d x \leq \int_{\mathbb{R} \times(0,1) \times \mathbb{R}}|h|^{2} d x .
$$

We now present the proofs of Theorems 2 and 3 .

Proof of Theorem 2. We proceed in several steps:

Step 1. We show that

$$
m_{1, k}-m_{1, \infty} \rightarrow 0 \quad \text { in } L^{1}(\mathbb{R}) \text { as } k \rightarrow \infty
$$

Indeed, from (1) and (15), we deduce that

$$
\begin{aligned}
\int_{\mathbb{R}}\left|m_{1, k}-m_{1, \infty}\right|^{2} d t & =\int_{-1}^{1}\left|m_{1, k}-m_{1, \infty}\right|^{2} d t \\
& =\int_{-1}^{1} \int_{2}^{3}\left|m_{1, k}(t)-m_{1, k}(t+s)\right|^{2} d t d s \\
& \leq 9 \int_{-1}^{1} \int_{2}^{3} \frac{\left|m_{1, k}(t)-m_{1, k}(t+s)\right|^{2}}{s^{2}} d t d s \\
& \leq 9 \int_{\mathbb{R}} \int_{\mathbb{R}} \frac{\left|m_{1, k}(t)-m_{1, k}(s)\right|^{2}}{|t-s|^{2}} d t d s \rightarrow 0 \quad \text { as } k \rightarrow \infty,
\end{aligned}
$$

and the conclusion follows by (1).

Step 2. We locate the regions where $m_{1, k}\left(\right.$ and $\left.m_{2, k}\right)$ have large variations. For that, we choose the intervals $\left(a_{n}^{k}, b_{n}^{k}\right), n=1, \ldots, N_{k}$, in the following way (see Figure 16): We set $b_{0}^{k}=-\infty$ and we recursively define, for $n=1, \ldots, N_{k}, b_{n}^{k} \in\left(b_{n-1}^{k}, 1\right]$ to be the smallest number such that

$$
m_{2, k}\left(b_{n}^{k}\right)=\frac{(-1)^{n-1} \sqrt{1-m_{1, \infty}^{2}}}{2}
$$




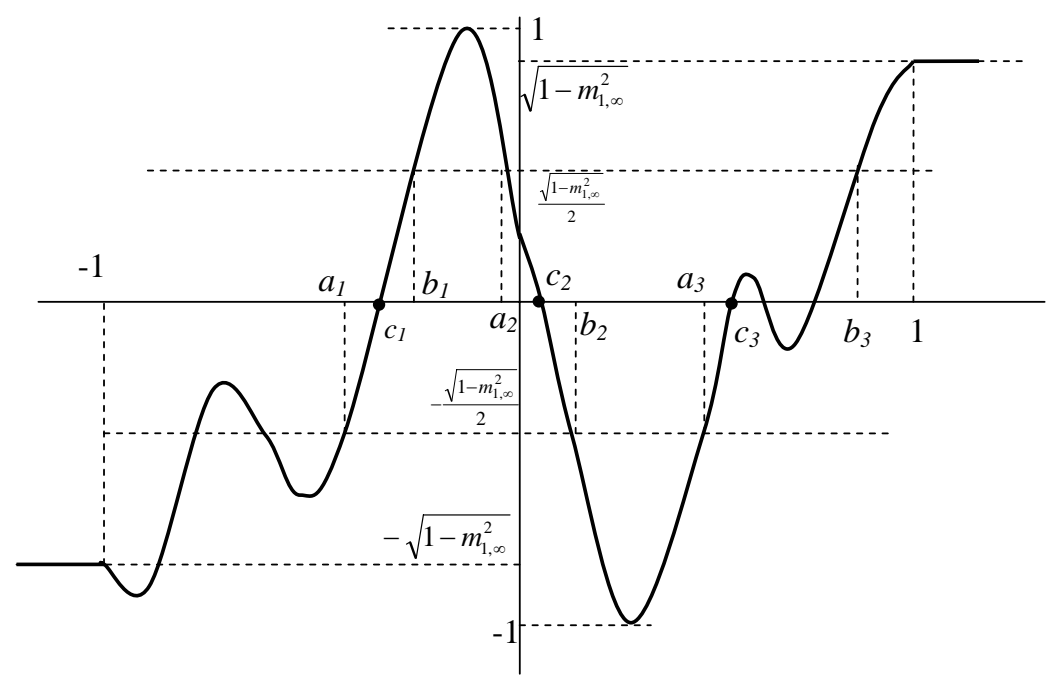

Fig. 16. The variations of $m_{2}$.

and respectively, $a_{n}^{k} \in\left[b_{n-1}^{k}, b_{n}^{k}\right]$ to be the largest number such that

$$
m_{2, k}\left(a_{n}^{k}\right)=\frac{(-1)^{n} \sqrt{1-m_{1, \infty}^{2}}}{2}
$$

By (1), we have

$-1<a_{1}^{k}<b_{1}^{k} \leq a_{2}^{k}<b_{2}^{k} \leq \cdots \leq a_{N_{k}}^{k}<b_{N_{k}}^{k}<1$ and $N_{k} \leq \frac{2}{1-m_{1, \infty}^{2}} \int_{\mathbb{R}}\left|\frac{d m_{2, k}}{d t}\right|^{2} d t$.

Indeed,

$$
\frac{1-m_{1, \infty}^{2}}{b_{n}^{k}-a_{n}^{k}}=\frac{1}{b_{n}^{k}-a_{n}^{k}}\left(\int_{a_{n}^{k}}^{b_{n}^{k}} \frac{d m_{2, k}}{d t} d t\right)^{2} \leq \int_{a_{n}^{k}}^{b_{n}^{k}}\left|\frac{d m_{2, k}}{d t}\right|^{2} d t \leq \int_{\mathbb{R}}\left|\frac{d m_{2, k}}{d t}\right|^{2} d t,
$$

and therefore

$$
N_{k} \leq \frac{\sum_{n=1}^{N_{k}}\left(b_{n}^{k}-a_{n}^{k}\right)}{1-m_{1, \infty}^{2}} \int_{\mathbb{R}}\left|\frac{d m_{2, k}}{d t}\right|^{2} d t \leq \frac{2}{1-m_{1, \infty}^{2}} \int_{\mathbb{R}}\left|\frac{d m_{2, k}}{d t}\right|^{2} d t
$$

We also notice that $N_{k}$ is an odd integer (by (1)), and

$$
\begin{gathered}
\left|m_{2, k}\right| \leq \sqrt{1-m_{1, \infty}^{2}} / 2 \quad \text { in any interval }\left(a_{n}^{k}, b_{n}^{k}\right), \\
(-1)^{n-1} m_{2, k} \leq \sqrt{1-m_{1, \infty}^{2}} / 2 \quad \text { in }\left(b_{n-1}^{k}, b_{n}^{k}\right), n=1, \ldots, N_{k} .
\end{gathered}
$$


Step 3. We prove that the sequence $\left\{N_{k}\right\}_{k \uparrow \infty}$ is bounded. The idea is to define a good step function with $2 N_{k}$ jumps and to apply Corollary 4. Set

$$
\chi_{k}= \begin{cases}\operatorname{sgn}\left(m_{1, k}\right) & \text { in }\left(a_{n}^{k}, c_{n}^{k}\right) \text { for } n=1, \ldots, N_{k}, \\ 0 & \text { elsewhere, }\end{cases}
$$

where $c_{n}^{k} \in\left[a_{n}^{k}, b_{n}^{k}\right]$ is the smallest number such that $m_{2, k}\left(c_{n}^{k}\right)=0$. Since (138) implies that $m_{1, k}$ does not change sign in $\left(a_{n}^{k}, c_{n}^{k}\right)$, we obtain:

$$
\begin{aligned}
\int_{\mathbb{R}}\left|\frac{d \chi_{k}}{d t}\right| & =2 N_{k}, \\
\int_{-1}^{1} \chi_{k} \frac{d m_{1, k}}{d t} d t & =\sum_{n=1}^{N_{k}} \int_{a_{n}^{k}}^{c_{n}^{k}} \operatorname{sgn}\left(m_{1, k}\right) \frac{d m_{1, k}}{d t} d t \\
& =\sum_{n=1}^{N_{k}}\left(\left|m_{1, k}\right|\left(c_{n}^{k}\right)-\left|m_{1, k}\right|\left(a_{n}^{k}\right)\right)=N_{k}\left(1-\frac{\sqrt{3+m_{1, \infty}^{2}}}{2}\right) .
\end{aligned}
$$

Now we apply Corollary 4 for the harmonic extension $U_{k}$ given by (136) associated to $m_{k}^{\prime}$ and for the test function $\eta=\eta\left(x_{1}, x_{3}\right): \mathbb{R}^{3} \rightarrow[-1,1]$ satisfying 1117 :

$$
\begin{aligned}
& \left|\int_{\mathbb{R} \times[0,1)} \eta^{2} \chi_{k} \frac{d m_{1, k}}{d x_{1}} d x^{\prime}\right| \leq\left(\frac{4}{\pi}\left|\ln \varepsilon_{k}\right| \int_{\mathbb{R} \times[0,1)} \eta^{2}\left|D^{\prime} \chi_{k}\right| \int_{\mathbb{R} \times[0,1) \times \mathbb{R}} \eta^{2}\left|\nabla U_{k}\right|^{2} d x\right)^{1 / 2} \\
& \quad+C\left(\varepsilon_{k} \int_{\mathbb{R} \times[0,1)}\left|\frac{d m_{1, k}}{d x_{1}}\right|^{2} d x^{\prime}+\int_{\mathbb{R} \times[0,1) \times \mathbb{R}}\left|\nabla U_{k}\right|^{2} d x\right)^{1 / 2}\left(1+\int_{\mathbb{R} \times[0,1)}\left|D^{\prime} \chi_{k}\right|\right),
\end{aligned}
$$

that is,

$$
\begin{aligned}
\left|\int_{-1}^{1} \chi_{k} \frac{d m_{1, k}}{d t} d t\right| \stackrel{137}{\leq} & C\left(\left|\ln \varepsilon_{k}\right| E_{\varepsilon_{k}}^{1 d}\left(m_{k}^{\prime}\right) \int_{\mathbb{R}}\left|\frac{d \chi_{k}}{d t}\right|\right)^{1 / 2} \\
& +\frac{C}{\sqrt{\left|\ln \varepsilon_{k}\right|}}\left(\left|\ln \varepsilon_{k}\right| E_{\varepsilon_{k}}^{1 d}\left(m_{k}^{\prime}\right)\right)^{1 / 2}\left(1+\int_{\mathbb{R}}\left|\frac{d \chi_{k}}{d t}\right|\right) .
\end{aligned}
$$

Therefore, by (15), (140) and (141), we deduce that $N_{k} \leq C$ for some absolute constant $C>0$.

Step 4. We show that the sequence $\left\{m_{2, k}\right\}_{k \uparrow \infty}$ is relatively compact in $L_{\text {loc }}^{1}$. We consider the step function

$$
\psi_{k}=\sum_{n=1}^{N_{k}+1}(-1)^{n} \sqrt{1-m_{1, \infty}^{2}} 1_{\left(b_{n-1}^{k}, b_{n}^{k}\right)}
$$

where $b_{N_{k}+1}^{k}=+\infty$. Observe that

$$
\int_{\mathbb{R}}\left|\frac{d \psi_{k}}{d t}\right|=2 N_{k} \sqrt{1-m_{1, \infty}^{2}}
$$


By Step 3, the sequence $\left\{\psi_{k}\right\}$ is bounded in $B V_{\text {loc }}(\mathbb{R})$. Therefore, any accumulation point $\psi: \mathbb{R} \rightarrow\left\{ \pm \sqrt{1-m_{1, \infty}^{2}}\right\}$ of $\left\{\psi_{k}\right\}_{k \uparrow \infty}$ in $L_{\text {loc }}^{1}$ is of bounded variation and has the form

$$
\psi=\sum_{n=1}^{2 N}(-1)^{n} \sqrt{1-m_{1, \infty}^{2}} 1_{\left(b_{n-1}, b_{n}\right)}
$$

where $-\infty=b_{0}<b_{1}<\cdots<b_{2 N-1}<b_{2 N}=+\infty$ and $b_{n} \in[-1,1]$ for $n=$ $1, \ldots, 2 N-1$. Finally, by 139 , we have

$$
\left|\psi_{k}+m_{2, k}\right| \geq \sqrt{1-m_{1, \infty}^{2}} / 2 \text { in } \mathbb{R},
$$

and therefore

$$
\begin{aligned}
\int_{\mathbb{R}}\left|\psi_{k}-m_{2, k}\right| d t=\int_{-1}^{1}\left|\psi_{k}-m_{2, k}\right| d t & \leq \frac{142}{\leq} \\
& \leq \frac{2}{\sqrt{1-m_{1, \infty}^{2}}} \int_{-1}^{1}\left|\psi_{k}^{2}-m_{2, k}^{2}\right| d t \\
& \leq \frac{2}{\sqrt{1-m_{1, \infty}^{2}}} \int_{-1}^{1}\left|\left(1-m_{1, \infty}^{2}\right)-m_{2, k}^{2}\right| d t \\
& \leq \frac{4}{\sqrt{1-m_{1, \infty}^{2}}} \int_{-1}^{1}\left|m_{1, k}-m_{1, \infty}\right| d t
\end{aligned}
$$

We conclude by Step 1 that up to a subsequence, $m_{2, k}-\psi \rightarrow 0$ in $L^{1}(\mathbb{R})$, i.e.,

$$
m_{k}^{\prime}-\left(\begin{array}{c}
m_{1, \infty} \\
\psi
\end{array}\right) \rightarrow 0 \quad \text { in } L^{1}(\mathbb{R}) \text { as } k \rightarrow \infty
$$

Since the asymptotic limits of the sequence $\left\{m_{k}^{\prime}\right\}_{k \uparrow \infty}$ in $L_{\text {loc }}^{1}$ belong to $B V$, one may ask whether the sequence $\left\{m_{k}^{\prime}\right\}$ is bounded in $B V$. The answer is negative according to Theorem 3 . The idea is that $m_{k}^{\prime}$ may have small variations on a large number of intervals (that have not been taken into account in the construction of the test functions $\chi_{k}$ in the previous proof).

Proof of Theorem 3. For simplicity, we assume that $m_{1, \infty}=0$. Set $\delta=\varepsilon^{1 / 4}, \omega=\varepsilon^{1 / 2}$ and $\eta=\varepsilon|\ln \varepsilon|$. For small $\varepsilon>0$, we consider the following function in $(-\omega, \omega)$ :

$$
f_{\varepsilon}(t)= \begin{cases}\frac{\delta}{|\ln \varepsilon|} \ln \frac{\omega}{\sqrt{t^{2}+\varepsilon^{2}}} & \text { if }|t| \leq \sqrt{\omega^{2}-\varepsilon^{2}}, \\ 0 & \text { if } t \in(-\omega, \omega) \backslash\left(-\sqrt{\omega^{2}-\varepsilon^{2}}, \sqrt{\omega^{2}-\varepsilon^{2}}\right) .\end{cases}
$$

This type of function was already used in [5]. We define $m_{1, \varepsilon}$ in $\mathbb{R}$ as follows: We fill in the intervals $(-1,-1 / 2)$, respectively $(1 / 2,1)$ by at most $1 / 4 \omega$ samples of $f_{\varepsilon}$ of length 
$2 \omega$ where $m_{1, \varepsilon}$ is given via $f_{\varepsilon}$, so that $m_{1, \varepsilon}$ is symmetric with respect to 0 . In the interval $\left(-\sqrt{1 / 2-\eta^{2}}, \sqrt{1 / 2-\eta^{2}}\right)$, set

$$
m_{1, \varepsilon}(t)=\frac{1}{|\ln (\sqrt{2} \eta)|} \ln \frac{1}{\left.\sqrt{2\left(t^{2}+\eta^{2}\right.}\right)} .
$$

Otherwise, we set $m_{1, \varepsilon}=0$. Hence, $m_{1, \varepsilon}$ is an even function in $H^{1}(\mathbb{R}), 0 \leq m_{1, \varepsilon} \leq \delta / 2$ in $\mathbb{R} \backslash(-1 / 2,1 / 2)$ and $m_{1, \varepsilon}(0)=1$. We then define

$$
m_{2, \varepsilon}(t)= \pm \sqrt{1-m_{1, \varepsilon}^{2}(t)} \quad \text { if } \pm t \geq 0
$$

hence, $m_{2, \varepsilon} \in H_{\mathrm{loc}}^{1}(\mathbb{R})$ and $(1)$ is satisfied. We compute the energy $E_{\varepsilon}^{1 d}\left(m_{1, \varepsilon}, m_{2, \varepsilon}\right)$. For $\varepsilon \ll 1$, we have

$$
\begin{aligned}
& \int_{(-1,-1 / 2) \cup(1 / 2,1)}\left|\frac{d m_{1, \varepsilon}}{d t}\right|^{2} d t \leq \frac{C}{\omega} \int_{-\omega}^{\omega} \frac{\delta^{2}}{|\ln \varepsilon|^{2}} \frac{t^{2}}{\left(t^{2}+\varepsilon^{2}\right)^{2}} d t \\
& \leq \frac{C}{\varepsilon|\ln \varepsilon|^{2}} \int_{0}^{\omega / \varepsilon} \frac{y^{2}}{\left(y^{2}+1\right)^{2}} d y \leq \frac{C}{\varepsilon|\ln \varepsilon|^{2}}\left(\int_{0}^{1} d y+\int_{1}^{\varepsilon^{-1 / 2}} \frac{d y}{y^{2}}\right) \leq \frac{C}{\varepsilon|\ln \varepsilon|^{2}} .
\end{aligned}
$$

Similarly, we compute that

$$
\int_{(-1 / 2,1 / 2)}\left|\frac{d m_{1, \varepsilon}}{d t}\right|^{2} d t \leq \frac{C}{\eta|\ln \eta|^{2}} \int_{0}^{\infty} \frac{y^{2}}{\left(y^{2}+1\right)^{2}} d y \leq \frac{C}{\varepsilon|\ln \varepsilon|^{3}} .
$$

Now we compute the homogeneous $H^{1 / 2}$-norm of $m_{1, \varepsilon}$. For that, we extend $m_{1, \varepsilon}$ to $\mathbb{R}^{2}$ by

$$
\tilde{m}_{1, \varepsilon}(t, s)=m_{1, \varepsilon}\left(\sqrt{t^{2}+s^{2}}\right), \quad \forall(t, s) \in \mathbb{R}^{2} .
$$

According to the trace estimate in $H^{1 / 2}$, it follows by the same argument as in (143) that

$$
\begin{aligned}
\int_{\mathbb{R}} \|\left.\left.\frac{d}{d t}\right|^{1 / 2} m_{1, \varepsilon}\right|^{2} d t & \leq \frac{1}{2} \int_{\mathbb{R}^{2}}\left|\nabla \tilde{m}_{1, \varepsilon}(t, s)\right|^{2} d t d s \\
& \leq \frac{C}{\omega} \int_{0}^{\omega} \frac{\delta^{2}}{|\ln \varepsilon|^{2}} \frac{t^{3}}{\left(t^{2}+\varepsilon^{2}\right)^{2}} d t+\frac{C}{|\ln \eta|^{2}} \int_{0}^{1 / 2} \frac{t^{3}}{\left(t^{2}+\varepsilon^{2}\right)^{2}} d t \\
& \leq \frac{C}{|\ln \varepsilon|}
\end{aligned}
$$

Hence, $|\ln \varepsilon| E_{\varepsilon}^{1 d}\left(m_{\varepsilon}^{\prime}\right) \leq C$ where $C>0$ is a universal constant. On the other hand, we have

$$
\begin{aligned}
\int_{\mathbb{R}}\left|\frac{d m_{1, \varepsilon}}{d t}\right| d t & \geq \int_{(-1,-1 / 2) \cup(1 / 2,1)}\left|\frac{d m_{1, \varepsilon}}{d t}\right| d t \\
& \geq \frac{C \delta}{\omega|\ln \varepsilon|} \int_{0}^{\omega} \frac{t}{t^{2}+\varepsilon^{2}} d t \geq \frac{C}{\varepsilon^{1 / 4}} \rightarrow \infty \quad \text { as } \varepsilon \rightarrow 0 .
\end{aligned}
$$

Acknowledgments. Part of this research was done during visits of Radu Ignat at the Institute of Applied Mathematics in Bonn, in the framework of the RTN Program "Fronts-Singularities"; he thanks for the invitation and hospitality. Felix Otto acknowledges partial support by the DFG through SFB 611 


\section{References}

[1] Abramowitz, M., Stegun, I. A.: Handbook of Mathematical Functions with Formulas, Graphs, and Mathematical Tables. National Bureau of Standards Appl. Math. Ser. 55, Washington, D.C. (1964) Zbl 0171.38503 MR 0167642

[2] Alouges, F., Rivière, T., Serfaty, S.: Néel and cross-tie wall energies for planar micromagnetic configurations. ESAIM Control Optim. Calc. Var. 8, 31-68 (2002) Zbl 1092.82047 MR 1932944

[3] Ambrosio, L., De Lellis, C., Mantegazza, C.: Line energies for gradient vector fields in the plane. Calc. Var. Partial Differential Equations 9, 327-255 (1999) Zbl 0960.49013 MR 1731470

[4] Coddington, E. A., Levinson, N.: Theory of Ordinary Differential Equations. McGraw-Hill, New York (1955) Zbl 0064.33002 MR 0069338

[5] DeSimone, A., Knüpfer, H., Otto, F.: 2-d stability of the Néel wall. Calc. Var. Partial Differential Equations 27, 233-253 (2006) Zbl pre05051269 MR 2251994

[6] DeSimone, A., Kohn, R., Müller, S., Otto, F.: A compactness result in the gradient theory of phase transitions. Proc. Roy. Soc. Edinburgh Sect. A 131, 833-844 (2001) Zbl 0986.49009 MR 1854999

[7] DeSimone, A., Kohn, R., Müller, S., Otto, F.: A reduced theory for thin-film micromagnetics. Comm. Pure Appl. Math. 55, 1408-1460 (2002) Zbl 1027.82042 MR 1916988

[8] García-Cervera, C. J.: One-dimensional magnetic domain walls. Eur. J. Appl. Math. 15, 451486 (2004) Zbl 1094.82019 MR 2115468

[9] Jabin, P.-E., Otto, F., Perthame, B.: Line-energy Ginzburg-Landau models: zero-energy states. Ann. Scuola Norm. Sup. Pisa Cl. Sci. (5) 1, 187-202 (2002) Zbl 1072.35051 MR 1994807

[10] Jin, W., Kohn, R. V.: Singular perturbation and the energy of folds. J. Nonlinear Sci. 10, 355390 (2000) Zbl 0973.49009 MR 1752602

[11] Melcher, Ch.: The logarithmic tail of Néel walls. Arch. Ration. Mech. Anal. 168, 83-113 (2003) Zbl pre02021055 MR 1991988

[12] Melcher, Ch.: Logarithmic lower bounds for Néel walls. Calc. Var. Partial Differential Equations 21, 209-219 (2004) Zbl 1054.78011 MR 2085302

[13] Riedel, R., Seeger, A.: Micromagnetic treatment of Néel walls. Phys. Statist. Sol. (B) 46, 377-384 (1971)

[14] Rivière, T., Serfaty, S.: Compactness, kinetic formulation, and entropies for a problem related to micromagnetics. Comm. Partial Differential Equations 28, 249-269 (2003) Zbl 1094.35125 MR 1974456 